TI 2015-084/III

Tinbergen Institute Discussion Paper
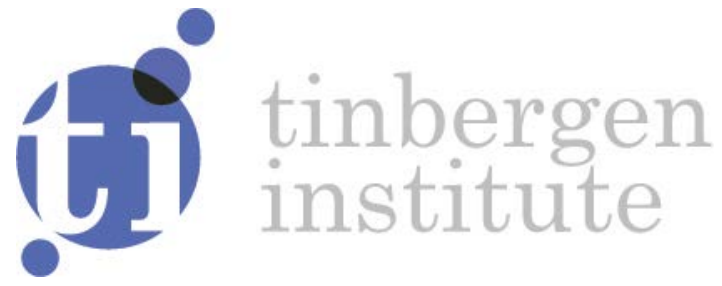

\title{
Dynamic Predictive Density Combinations for Large Data Sets in Economics and Finance
}

Roberto Casarin ${ }^{1}$

Stefano Grassi ${ }^{2}$

Francesco Ravazzolo ${ }^{3}$

Herman K. van Dijk ${ }^{4}$

${ }^{1}$ University Ca' Foscari of Venice, Italy

${ }^{2}$ University of Kent, United Kingdom

${ }^{3}$ Norges Bank, Norway

${ }^{4} \mathrm{VU}$ University Amsterdam, Erasmus University Rotterdam, the Netherlands 
Tinbergen Institute is the graduate school and research institute in economics of Erasmus University Rotterdam, the University of Amsterdam and VU University Amsterdam.

Contact: discussionpapers@tinbergen.nl

More TI discussion papers can be downloaded at http://www.tinbergen.nl

Tinbergen Institute has two locations:

Tinbergen Institute Amsterdam

Gustav Mahlerplein 117

1082 MS Amsterdam

The Netherlands

Tel.: $+31(0) 205984580$

Tinbergen Institute Rotterdam

Burg. Oudlaan 50

3062 PA Rotterdam

The Netherlands

Tel.: +31(0)104088900 


\title{
Dynamic Predictive Density Combinations for Large Data Sets in Economics and Finance*
}

\author{
Roberto Casarin ${ }^{\dagger} \quad$ Stefano Grassi $i^{\ddagger}$ \\ Francesco Ravazzolo ${ }^{\S}$ Herman K. van Dijk $₫$ \\ †University Ca’ Foscari of Venice \\ †University of Rome 'Tor Vergata' \\ $\S$ Free University of Bozen/Bolzano \\ IEconometric Institute, Erasmus University Rotterdam, Norges Bank \\ and Tinbergen Institute \\ March 2017 \\ First draft: July 2015
}

\begin{abstract}
A Bayesian semi-parametric dynamic model combination is proposed in order to deal with a large set of predictive densities. It extends the

\footnotetext{
${ }^{*}$ We thank John Geweke, Jim Stock, Peter Schotman, Peter Hansen, Gael Martin, Michael Smith, Gernot Doppelhofer, Anastasios Panagiotalis, Barbara Rossi and conference and seminar participants at Erasmus University Rotterdam Workshop on "The Econometric Analysis of Recurrent Events in Macroeconomics and Finance", the 34th International Symposium on Forecasting, the 8th International CFE meeting in Pisa, the 25th EC ${ }^{2}$ Conference on "Advances in Forecasting", the RCEA 9th Rimini Bayesian Workshop, the EABCN Conference at Norges Bank, the IAAE Conference in Thessaloniki, Free University of Bozen-Bolzano, Institute for Advance Studies Vienna, Maastricht University, Monash University, Norges Bank, the Stevanovich Center at University of Chicago, UTS Sydney, and UPF Barcelona, for very useful comments. Roberto Casarin's research is supported by funding from the European Union, Seventh Framework Programme FP7/2007-2013 under grant agreement SYRTO-SSH-2012-320270, by the Institut Europlace of Finance, "Systemic Risk grant", the Global Risk Institute in Financial Services, the Louis Bachelier Institute, "Systemic Risk Research Initiative", and by the Italian Ministry of Education, University and Research (MIUR) PRIN 2010-11 grant MISURA.
} 
mixture of experts and the smoothly mixing regression models by allowing combination weight dependence between models as well as over time. It introduces an information reduction step by using a clustering mechanism that allocates the large set of predictive densities into a smaller number of mutually exclusive subsets. The complexity of the approach is further reduced by making use of the class-preserving property of the logisticnormal distribution that is specified in the compositional dynamic factor model for the weight dynamics with latent factors defined on a reduced dimension simplex. The whole model is represented as a nonlinear state space model that allows groups of predictive models with corresponding combination weights to be updated with parallel clustering and sequential Monte Carlo filters. The approach is applied to predict Standard \& Poor's 500 index using more than 7000 predictive densities based on US individual stocks and finds substantial forecast and economic gains. Similar forecast gains are obtained in point and density multivariate combined forecasts of US real GDP, Inflation, Treasury Bill yield and employment using a large data set. The dynamic patterns in the clusterbased weights provide valuable signals for improved economic modeling and forecasting.

JEL codes: C11, C15, C53, E37.

Keywords: Density Combination, Large Set of Predictive Densities, Compositional Factor Models, Nonlinear State Space, Bayesian Inference, GPU Computing.

\section{Introduction}

Forecasting with large sets of data is a topic of substantial interest to academic researchers as well as to professional and applied forecasters. It has been studied in several papers (e.g., see Stock and Watson, 1999, 2002, 2005, 2014, and Bańbura et al., 2010). The recent fast growth in (real-time) big data allows researchers to predict variables of interest more accurately (e.g., see Choi and 
Varian, 2012; Varian, 2014; Varian and Scott, 2014; Einav and Levin, 2014).

Stock and Watson (2005, 2014), Bańbura et al. (2010) and Koop and Korobilis (2013) suggest, for instance, that there are potential gains from forecasting using a large set of predictors instead of a single predictor from a univariate time series. However, forecasting with many predictors and highdimensional models requires new modeling strategies (to keep the number of parameters and latent variables relatively small), efficient inference methods and extra computing power like parallel computing. We refer to Granger (1998) for an early discussion of these issues.

We propose a Bayesian semi-parametric dynamic model combination in order to deal with a large set of predictive densities. Our model extends the mixture of the experts and the smoothly mixing regression models (Jacobs et al., 1991, Jordan and Jacobs, 1994, Jordan and Xu, 1995, Peng et al., 1996, Wood et al., 2002, Geweke and Keane, 2007, Villani et al., 2009, Norets, 2010) by allowing the combination weights to be dependent between models as well as over time and to further allow for model incompleteness. We introduce an information reduction step by using a clustering mechanism that allocates the large set of predictive densities into a smaller number of mutually exclusive subsets. The complexity of the approach is further reduced by making use of the class-preserving property of the logistic-normal distribution that is specified in a compositional dynamic factor model for the weight dynamics where the latent factors are defined on a reduced dimension simplex. Here the class-preserving property of the logisticnormal distribution (see Aitchinson and Shen, 1980, Aitchinson, 1982) is used. The whole model is represented as a nonlinear state space model that allows groups of predictive models with corresponding combination weights to be 
updated with parallel clustering and sequential Monte Carlo filters. We also show that, given that the space of the weight measures is equipped with suitable operations and norms, this nonlinear state space model may be interpreted as a generalized linear model with a local level component. In this respect, our approach contributes to the literature on time series on a bounded domain (see, e.g., Aitchinson, 1982, Aitchinson, 1986 and Billheimer et al., 2001) and on state space models for compositional data analysis (see, e.g., Grunwald et al., 1993). In that literature the compositional data are usually observed, while in our model the weights are latent probabilities.

Finally, we propose a Bayesian diagnostic analysis to indicate particular types of missing information and use De Finetti's graphs to indicate the important evolution patterns in the model weights, see also Ehm et al. (2016) for the use of graphical analysis for forecast evaluation. The parallelized sequential prediction and filtering methods to efficiently update the dynamic clustered weights of the combination model are made operational by following the recent trend of using graphics processing units (GPU) for general, non-graphics, applications, the socalled general-purpose computing on GPU (GPGPU).

The proposed method is applied to two well-known problems in economics and finance. In the first macro-finance example, we find substantial gains in point and density multivariate forecasts of US real GDP, GDP deflator inflation, Treasury Bill yield and employment over the last 25 years for all horizons from one-quarter ahead to five-quarter ahead. The highest accuracy is achieved when the four series are predicted simultaneously using our combination schemes within and across cluster weights based on log score learning. A dominant set of variables does not exist but we note that the cluster that includes Exports, 
Imports and GDP deflator receives a large weight. In the second example we use more than 7000 predictive densities based on 3712 US individual stock return series to replicate the daily aggregate S\&P 500 returns over the sample 2007-2009 and predict the economic value of tail events like Value-at-Risk. The method allows for time-varying composition of the clusters letting individual stocks to switch across them or eventually exit the model set, for example after a default, as in the Lehman Brothers case. We find large accuracy gains in all distribution and also in the tail analysis with respect to the no-predictability benchmark and predictions from individual models estimated on the aggregate index that result in violations of the Value-at-Risk very similar to the nominal value. The observed dynamic patterns in the cluster-based weights provide valuable signals for improved economic/financial modeling and forecasting.

The contents of this paper is structured as follows. Sections 2 and 3 provide details of the contributions of our approach. Section 4 applies our model to Stock and Watson (2005) macroeconomic data set and a large set of US stocks. Section 5 contains conclusions and suggestions for further research. The Supplementary Material contains more details on data, derivations and results.

\section{Mixtures, information reduction and sequential clustering using large data sets}

There exists an increasing interest in the recent literature about combinations of predictive densities with possibly time-varying weights, see, e.g., Billio et al. (2013) and Casarin et al. (2015). In this context Waggoner and Zha 
(2012) and Del Negro et al. (2015) propose time-varying weights in the linear opinion framework and Fawcett et al. (2015) introduce a similar concept in the generalized linear pool. Conflitti et al. (2015) propose optimal combinations of large sets of point and density survey forecasts; their weights are, however, not modeled with time-varying patterns, while Raftery et al. (2010) develop dynamic model averaging that allows the "correct" model to vary over time.

Our contributions are summarized as follows. We extend the standard density combination approach, which consists of a model combination density, a weight density and a density of the predictors of many models, to a large finite mixture of convolutions of densities where the mixture weights are dependent over time and between models. The dependence between models seems quite important in this framework considering the large correlations of point and density forecasts, but it has not been formally treated in density forecasting. A second contribution is to reduce the complexity of our approach by introducing an information reduction step that uses a clustering mechanism that allocates the large set of predictive densities in much smaller mutually exclusive subsets. In the information reduction step the class-preserving property of the logisticnormal distribution is used. This extension allows to combine several gigabyte of data with a reasonable computational effort. A third contribution is the dynamic specification of the latent cluster specific weights in a compositional factor model which evolves over time and allows for learning. Here we contribute to the literature of time series on a bounded domain. The contributions listed so far allow us to represent the complete model as a nonlinear state space model where the measurement equation refers to the combination model and the nonlinear transition function of the latent weights is a dynamic compositional factor model 
with a noise process that follows a multivariate logistic-normal distribution.

\subsection{A large dynamic mixture of convolutions of densities as expert system}

Let $\mathbf{y}_{t}=\left(y_{1 t}, \ldots, y_{K t}\right)^{\prime}$ be the $K$-dimensional vector of variables of interest, and $\tilde{\boldsymbol{y}}_{t}=\left(\tilde{y}_{1 t}, \ldots, \tilde{y}_{n t}\right)^{\prime}$ be a vector of a large set of $n$ random predictors for the variables of interest with densities $f_{i t}\left(\tilde{y}_{i t}\right)$, from $i=1, \ldots, n$ economic and/or financial models, with each prediction conditional on the information set available at time $t-1$. Let $\mathbf{w}_{k t}=\left(w_{1, k t}, \ldots, w_{n, k t}\right)^{\prime}$ be a set of random weights. In the next subsection a process for these random weights is specified, which refers to a learning function based on past predictive performance of models.

At time $t-1$, we assume a sequence of Gaussian conditional predictive densities is available

$$
f\left(y_{k t} \mid \tilde{y}_{i t}, \sigma_{k t}^{2}\right)
$$

$k=1, \ldots, K$. Then we can specify a large finite Gaussian mixture combination model

$$
f\left(y_{k t} \mid \mathbf{w}_{k t}, \tilde{\boldsymbol{y}}_{t}, \sigma_{k t}^{2}\right) \sim \sum_{i=1}^{n} w_{i, k t} f\left(y_{k t} \mid \tilde{y}_{i t}, \sigma_{k t}^{2}\right)
$$

$k=1, \ldots, K, t=1, \ldots, T$, where $\sigma_{k t}^{2}, t=1, \ldots, T$, is defined to follow the stochastic volatility process

$$
\log \sigma_{k t}^{2} \sim f\left(\log \sigma_{k t}^{2} \mid \log \sigma_{k, t-1}^{2}, \sigma_{\eta_{k}}^{2}\right)
$$


The process $\sigma_{k t}^{2}$ controls the overall uncertainty level about the prediction models used in the combination. We emphasize that in our approach this overall level of uncertainty is a major indicator of incompleteness of the set of predictive models. Indeed, when the value of $\sigma_{k t}^{2}$ is large, the overall uncertainty and the misspecification of each predictors are significant. On the contrary, when the uncertainty level tends to zero then we recover as a limiting case the mixture of experts or the smoothly mixing regressions models.

Given these specifications, one can derive the following proposition.

Proposition 2.1 (Mixture representation). Under standard regularity conditions and given the conditional information sets of all individual models, the marginal predictive density of $y_{k t}$ has the following discrete/continuous mixture representation

$$
f\left(y_{k t} \mid \mathbf{w}_{k t}, \sigma_{k t}^{2}\right)=\sum_{i=1}^{n} w_{i, k t} \int_{\mathbb{R}} f\left(y_{k t} \mid \tilde{y}_{i t}, \sigma_{k t}^{2}\right) f_{i t}\left(\tilde{y}_{i t}\right) d \tilde{y}_{i t}, \quad k=1, \ldots, K
$$

If the overall uncertainty level, controlled by $\sigma_{k t}^{2}$, tends to zero, then

$$
f_{k t}\left(y_{k t} \mid \mathbf{w}_{k t}\right) \longrightarrow \sum_{i=1}^{n} w_{i, k t} f_{i t}\left(y_{k t}\right), k=1, \ldots, K
$$

Proof, see Supplementary Material.

We started this section by stating that the analysis is conditional upon the information specified in the different models. Our method allows to study for which model or cluster of models, incompleteness will be larger over time and also to study the overall level of incompleteness. The importance of this measuring is shown in our empirical analyses. 
All the proposed extensions to the basic density combination model permit a proper Bayesian inference based on prior and posterior distributions of the large density combination scheme, including information reduction, timevarying weights over time, dependence across models, and model incompleteness. The next two subsections discuss specific parameters for these topics. The following section describes another contribution of this paper related to parallel computing. We make use of parallelized sequential prediction and filtering methods in order to update efficiently the dynamic clustered weights of the combination model.

\subsection{Information reduction and time varying weight specification}

In the specification of the combination model given in the previous section, the number of latent processes to estimate is $n K$ at every time period $t$ which can be computationally heavy, even when a small number of variables of interest,

e.g. 4, and a moderate number of models, e.g. $n=100$, are considered. A contribution of this paper is to diminish the complexity of the combination exercise by reducing the dimension of the latent space by preserving crucial aspects such as time-varying weights and model dependence.

As a first step, the $n$ predictors are clustered into $m$ different groups, with $m<n$, following some (time-varying) features $\boldsymbol{\psi}_{i t}, i=1, \ldots, n$, of the predictive densities. This allows to deal with model dependence, a feature well documented on data but often ignored in density forecasting. We introduce $\xi_{j, i t}$ as an allocation variable, which takes the value 1 if the $i$-th predictor is assigned to 
the $j$-th group of densities and 0 otherwise. We assume each predictor belongs to only one group, that is $\sum_{j=1}^{m} \xi_{j, i t}=1$ for all $i$. Therefore, models and/or predictions with similar level of dependence can be grouped together. Also, the grouping of the predictors can change over time, following a learning mechanism which is defined by a sequential clustering rule. In such a way, even if the number of clusters is kept constant over time, clustering varies. The number of clusters could also be considered to vary over time, but their interpretation would be very difficult and possible not valid. Details of the learning mechanism are presented later in this section and the sequential clustering rule is given in the following section.

Given the clustering of the predictors, we specify how to reduce the dimension of the latent weight space from $n K$ to $m K$ with $m<n$. To this aim, we specify the $(n \times m)$ allocation matrix $\Xi_{t}=\left(\boldsymbol{\xi}_{1 t}, \ldots, \boldsymbol{\xi}_{m t}\right)$, with $\boldsymbol{\xi}_{j t}=\left(\xi_{j, 1 t}, \ldots, \xi_{j, n t}\right)^{\prime}$, $j=1, \ldots, m$, the vector of allocation variables $\xi_{j, i t} \in\{0,1\}$, and a $(n \times m)$ coefficient matrix $B_{k t}$ with the $i$-th row and $j$-th column element given by $b_{i j, k t} \in \mathbb{R}$. The two matrices allow us to project the $n$-dimensional latent variable $\mathbf{x}_{k t}$ onto a reduced dimension latent space, through the following latent factor model

$$
\mathbf{x}_{k t}=\left(\Xi_{t} \circ B_{k t}\right) \mathbf{v}_{k t}
$$

where $\circ$ denotes the element-by-element Hadamard's product, and $\boldsymbol{v}_{k t}=$ $\left(v_{1, k t}, \ldots, v_{m, k t}\right)^{\prime}$ is a $m$-variate normal random walk process

$$
\boldsymbol{v}_{k t}=\boldsymbol{v}_{k, t-1}+\chi_{k t}, \quad \chi_{k t} \stackrel{i i d}{\sim} \mathcal{N}_{m}\left(\mathbf{0}_{m}, \Upsilon_{k}\right)
$$

The process $\boldsymbol{v}_{k t}, t=1, \ldots, T$, is latent and is driving the weights of the predictive 
densities which are used to forecast the $k$-th variable of interest. The set of all variable-specific latent processes, is associated width a latent space of dimension $m K$. The coefficients, $\xi_{j, i t}$ and $b_{i j, k t}, j=1, \ldots, m$, for each variable of interest $k$, predictor $j$ and time $t$, are crucial in order to obtain a parsimonious latent variable model and consequently to reduce the computational complexity of the combination procedure without sacrificing key features such as time-variation and model dependence.

For specific values of the coefficients $b_{i j, k t}$, we propose two alternative strategies. The first one is where all coefficients in the cluster have the same weights, which corresponds to set $b_{i j, k t}$ as:

$$
b_{i j, k t}= \begin{cases}1 / n_{j t} & \text { if } \xi_{j, i t}=1 \\ 0 & \text { otherwise }\end{cases}
$$

where

$$
n_{j t}=\sum_{i=1}^{n} \xi_{j, i t}
$$

is the number of predictive densities in the $j$-th cluster at time $t$. Note that, following this specification of the coefficients, the weights of the $n$ predictors for the $k$-th variable of interest are

$$
w_{i, k t}=\frac{\exp \left\{v_{j_{i}, k t} / n_{j_{i}}\right\}}{\sum_{j=1}^{m} \exp \left\{v_{j, k t} / n_{j t}\right\}}, \quad i=1, \ldots, n
$$

where $j_{i}=\sum_{j=1}^{m} j \xi_{j, i t}$ indicates the group to which the $i$-th predictor belongs. The latent weights are driven by a set of $m$ latent variables, with $m<n$, thus the dimensional reduction of the latent space is achieved. Moreover, let 
$N_{i t}=\left\{j=1, \ldots, n \mid \xi_{i, j t}=1\right\}$ be the set of the indexes of all models in the cluster $i$, then one can see that this specifications may have the undesirable property that the weights are constant within a group, that is for all $j \in N_{i t}$.

For this reason, we also propose a second specification strategy where we assume that each model contributes to the combination with a specific weight that is driven by a model-specific forecasting performance measure. Here the learning aspect appears. If we assume $g_{i t}$ is the log score (see definition in (S.26)) of the model $i$ at time $t$ then

$$
b_{i j, k t}= \begin{cases}\sum_{s=1}^{t} \exp \left\{g_{i s}\right\} / \bar{g}_{i t} & \text { if } \xi_{j, i t}=1 \\ 0 & \text { otherwise }\end{cases}
$$

where $\bar{g}_{i t}=\sum_{l \in N_{i t}} \sum_{s=1}^{t} \exp \left\{g_{l s}\right\}$.

All the modeling assumptions discussed above allow us to reduce the complexity of the combination exercise because the set of time-varying combination weights to estimate is of dimension $m K<<<n K$.

\subsection{Reduced-dimension state-space representation}

The density combination model proposed in this paper can be written in terms of a nonlinear state space model defined on a reduced-dimension latent space. Moreover, thanks to the class-preserving property of the logisticnormal distribution, the proposed transition density can be represented as a compositional latent factor model. We also show that this nonlinear state space model may be written in the form of a generalized linear model with a local level component when the space of the random measures is equipped with suitable 
operations and norms.

\subsubsection{Probabilistic information reduction}

We start to introduce some useful results and definitions. Let $\mathbb{S}^{n}=\{\mathbf{u} \in$ $\left.\mathbb{R}_{+}^{n} \mid u_{1}+\ldots+u_{n}<1\right\}$ be the $n$-dimensional standard simplex, where $\mathbb{R}_{+}^{n}$ denotes the positive orthant of $\mathbb{R}^{n}$. Proofs of results are presented in the Supplementary Material S.1.

Definition 2.1 (Composition function). The function $C_{m}(\mathbf{u}): \mathbb{R}_{+}^{m} \rightarrow \mathbb{S}^{m-1}$, $\mathbf{u} \mapsto \mathbf{v}=C_{m}(\mathbf{u})$ with the $i$-the element of $\mathbf{v}$ defined as $v_{i}=u_{i} / v_{m}, i=$ $1, \ldots, m-1$, with $v_{m}=\mathbf{u}^{\prime} \iota_{m}$.

Proposition 2.2 (Logistic-normal distribution). Let $\mathbf{v} \sim \mathcal{N}_{m}(\boldsymbol{\mu}, \Upsilon)$, and define $\mathbf{u}=\exp (\mathbf{v})$, that is the component-wise exponential transform of $\mathbf{v}$, and $\mathbf{z}=C_{m}(\mathbf{u})$, that is the composition of $\mathbf{u}$, then $\mathbf{u}$ follows a m-variate log-normal distribution, $\Lambda_{m}(\boldsymbol{\mu}, \Upsilon)$, and $\mathbf{z}$ follows a logistic-normal distribution $\mathcal{L}_{m-1}\left(D_{m} \boldsymbol{\mu}, D_{m} \Upsilon D_{m}^{\prime}\right)$ with density function

$$
\begin{gathered}
p(\mathbf{z} \mid \boldsymbol{\mu}, \Upsilon)=\left|2 \pi D_{m} \Upsilon D_{m}^{\prime}\right|^{-1 / 2}\left(\prod_{j=1}^{m-1} z_{j}\right)^{-1} \exp \left(-\frac{1}{2}\left(\log \left(\mathbf{z} / z_{m}\right)-D_{m} \boldsymbol{\mu}() 10\right)\right. \\
\left.\left(D_{m} \Upsilon D_{m}^{\prime}\right)^{-1}\left(\log \left(\mathbf{z} / z_{m}\right)-D_{m} \boldsymbol{\mu}\right)^{\prime}\right)
\end{gathered}
$$

where $\mathbf{z} \in \mathbb{S}^{m-1}, z_{m, k t}=1-\mathbf{z}^{\prime} \boldsymbol{\iota}_{m-1}, D_{m}=\left(I_{m-1},-\boldsymbol{\iota}_{m-1}\right)$ and $\boldsymbol{\iota}_{m-1}$ is the $(m-1)$ unit vector.

Corollary 2.1. Let $\mathbf{v}_{k t} \sim \mathcal{N}_{m}\left(\mathbf{v}_{k t-1}, \Upsilon_{k}\right)$, and $\mathbf{z}_{k t}=C_{m}\left(\exp \left(\mathbf{v}_{k t}\right)\right)$, then $\mathbf{z}_{k t} \in \mathbb{S}^{m-1}$ follows the logistic-normal distribution $\mathcal{L}_{m-1}\left(D_{m} \mathbf{v}_{k t-1}, D_{m} \Upsilon_{k} D_{m}^{\prime}\right)$. 
The class-preserving property of the composition of the logistic-normal vectors (see Aitchinson and Shen, 1980) will be used in the proof of the main result of this section. We show how this property adapts to our state space model.

Proposition 2.3 (Class-preserving property). Let $A a(c \times m-1)$ matrix and $\mathbf{z}_{k t} \sim \mathcal{L}_{m-1}\left(D_{m} \mathbf{v}_{k t-1}, D_{m} \Upsilon_{k} D_{m}^{\prime}\right)$ a logistic-normal vector. Define the following transform $\mathbf{w}=\phi_{A}(\mathbf{z})$ from $\mathbb{S}^{m-1}$ to $\mathbb{S}^{c}$, with in our case $m<c$,

$$
w_{i, k t}=\prod_{j=1}^{m-1}\left(\frac{z_{j, k t}}{z_{m, k t}}\right)^{a_{i j}}\left(1+\sum_{i=1}^{c} \prod_{j=1}^{m-1}\left(\frac{z_{j, k t}}{z_{m, k t}}\right)^{a_{i j}}\right)^{-1}
$$

$i=1, \ldots, c$, then $\mathbf{w}_{k t}=\left(w_{1, k t}, \ldots, w_{c, k t}\right)$ follows the logistic-normal $\mathcal{L}_{c}\left(A D_{m} \mathbf{v}_{k t-1}, A D_{m} \Upsilon_{k} D_{m}^{\prime} A^{\prime}\right)$.

\subsubsection{A reduced-dimension state-space representation}

Given the results in the preceding subsection, we can now state the main result.

Proposition 2.4 (State-space form). Let $A_{k t}=\Xi_{t} \circ B_{k t}, k=1, \ldots, K$, be a matrix of coefficients, then the model given in equations 2-6 can be written in the following state space form

$$
\begin{aligned}
\mathbf{y}_{t} & \sim \prod_{k=1}^{K} \sum_{i=1}^{n} w_{i, k t} \mathcal{N}\left(\tilde{y}_{i t}, \sigma_{k t}^{2}\right) \\
\tilde{\mathbf{w}}_{k t} & \sim \mathcal{L}_{n-1}\left(\tilde{\tilde{A}}_{k t} D_{m} \mathbf{v}_{k t-1}, \tilde{\tilde{A}}_{k t} D_{m} \Upsilon_{k} D_{m}^{\prime} \tilde{\tilde{A}}_{k t}^{\prime}\right), \quad k=1, \ldots, K
\end{aligned}
$$

$\tilde{\mathbf{w}}_{k t}=\left(w_{1, k t}, \ldots, w_{n-1, k t}\right)^{\prime}$ and $w_{n, k t}=1-\tilde{\mathbf{w}}_{k t}^{\prime} \boldsymbol{\iota}_{n-1}, \otimes$ denotes the Kronecker's product, $\tilde{\tilde{A}}_{k t}=\left(\tilde{A}_{k t}^{\prime}, O_{\left(n-\tilde{n}_{t}\right) \times(m-1)}^{\prime}\right)^{\prime}$, with $\tilde{n}_{t}=\operatorname{Card}\left(\tilde{N}_{t}\right)$ and $\tilde{N}_{t}=\{i=$ $\left.1, \ldots, n \mid \xi_{m, i t} \neq 1\right\}$ the set of indexes of the models allocated in the cluster $m$. 

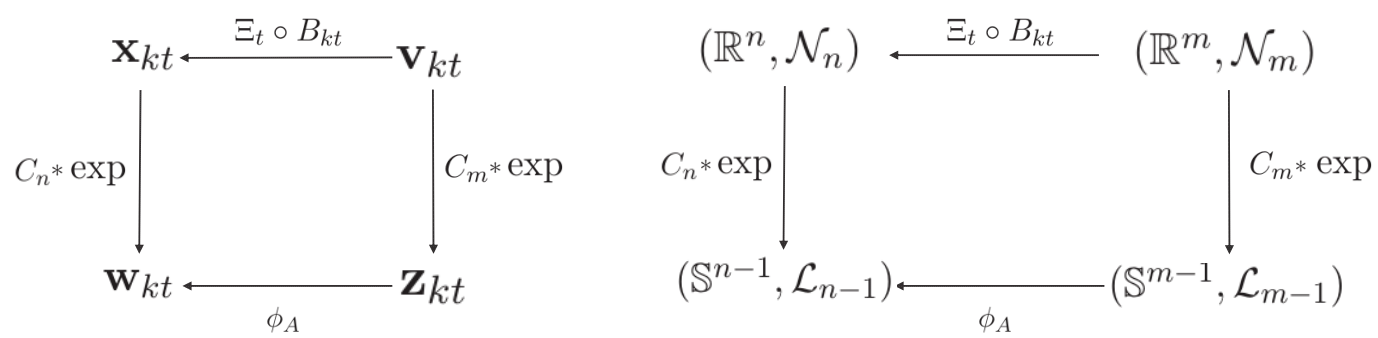

Figure 1: Relationships between the latent variables (left) and the latent probability spaces (right) involved in our compositional latent factor model. The origin of the directed edge indicates the transformed variable, the arrow indicates the results of the transformation, and the edge label defines the transform applied. The symbol $*$ indicates a composition of functions.

The previous proposition establishes a relationship between the set of latent weights $\mathbf{w}_{k t}$ and their projection, $\mathbf{z}_{k t}$, on the lower dimension latent space $\mathbb{S}^{m-1}$. The diagram on the left side of Figure 1 summarizes the relationships between the latent variables involved in our compositional latent factor model. The symbol $*$ indicates function composition. The diagram on the right shows the relationship between the probability latent spaces. In both diagrams, the chaining process given by the function composition $\phi_{A} * C_{m} * \exp$ indicates that the probabilistic interpretation of the $n$-dimensional weight vector $\mathbf{w}_{k t}$ naturally transfers to the $m$-dimensional vector $\mathbf{z}_{k t}$, with $m<n$.

In the same diagram an alternative chaining process is given by the function composition $C_{n} * \exp *\left(\Xi_{t} \circ B_{k t}\right)$, which allows for the following alternative representation of the latent factor model as a logistic-normal factor model.

Corollary 2.2. The transition density given in Proposition 2.4 can be written as $\tilde{\mathbf{w}}_{k t} \sim \mathcal{L}_{n-1}\left(D_{n} A_{k t} \mathbf{v}_{k t-1}, D_{n} A_{k t} D_{n} \Upsilon_{k} D_{n}^{\prime} A_{k t}^{\prime}\right)$ and $w_{n, k t}=1-\tilde{\mathbf{w}}_{k t}^{\prime} \iota_{n-1}$.

Distributions other than the logistic-normal can be used for weights such 
as the Dirichlet distribution, but as noted in Aitchinson and Shen (1980) this distribution may be too simple to be realistic in the analysis of compositional data since the components of a Dirichlet composition have a correlation structure determined solely by the normalization operation in the composition. See, Aitchinson and Shen (1980) for a complete discussion of the advantages of the logistic-normal distribution compared to the Dirichlet.

We also present another result that shows how the state space model can be written as a generalized linear model with a local level transition function when the space of the random measures is equipped with suitable operations and norms. Moreover, we show that the probabilistic interpretation is preserved for the lower dimensional set of latent weights.

Define the observation real space $\mathbb{R}^{K}$ equipped with the inner product $<\mathbf{x}, \mathbf{y}>=\sum_{i=1}^{K} x_{i} y_{i}, \mathbf{x}, \mathbf{y} \in \mathbb{R}^{K}$ and scalar product $a \mathbf{x}=\left(a x_{1}, \ldots, a x_{K}\right)^{\prime}$, $\mathbf{x} \in \mathbb{R}^{K}, a \in \mathbb{R}$ operations. Also, define the simplex (state) space, $\mathbb{S}^{n-1}$ equipped with a sum operation (also called perturbation operation), $\mathbf{u} \oplus \mathbf{v}=C(\mathbf{u} \circ \mathbf{v})$, $\mathbf{u}, \mathbf{v} \in \mathbb{S}^{n-1}$ and a scalar product operation (also called power transform) $a \odot \mathbf{u}=C\left(\left(u_{1}^{a}, \ldots, u_{n-1}^{a}\right)^{\prime}\right), \mathbf{u} \in \mathbb{S}^{n-1}, a \in \mathbb{R}_{+}$. For details and background, see Aitchinson (1986) and Aitchinson (1992). Billheimer et al. (2001) showed that $\mathbb{S}^{n-1}$ equipped with the perturbation and powering operations is a vector space. Moreover $\mathbb{S}^{n-1}$ is an Hilbert space, i.e. a complete, inner product vector space, equipped with the inner product $\langle\mathbf{u}, \mathbf{v}\rangle_{N}=\mathbf{u}, \mathbf{v} \in \mathbb{S}^{n-1}$ space. These properties enable us to state the following result.

Corollary 2.3. Let $\mathbf{s}_{t}=\left(\mathbf{s}_{1 t}^{\prime}, \ldots, \mathbf{s}_{K t}^{\prime}\right)^{\prime}$ be an allocation vector, with $\mathbf{s}_{k t} \sim$ $\mathcal{M}_{n}\left(1, \mathbf{w}_{k t}\right), \quad k=1, \ldots, K$, where $\mathcal{M}_{n}\left(1, \mathbf{w}_{k t}\right)$ denotes the multinomial distribution, and $\Sigma_{t}=\operatorname{diag}\left\{\sigma_{1 t}^{2}, \ldots, \sigma_{K t}^{2}\right\}$ a covariance matrix. Then, the state 
space model given in Proposition 2.4 can be written as

$$
\begin{aligned}
\mathbf{y}_{t} & =\left(I_{K} \otimes \tilde{\mathbf{y}}_{t}^{\prime}\right) \mathbf{s}_{t}+\boldsymbol{\varepsilon}_{t}, \quad \boldsymbol{\varepsilon}_{t} \sim \mathcal{N}_{K}\left(\mathbf{0}, \Sigma_{t}\right) \\
s_{i, k t} & =\left\{\begin{array}{rr}
1 & \text { with probability } w_{i, k t} \\
0 & \text { otherwise }
\end{array}\right. \\
\mathbf{w}_{t} & =\boldsymbol{\phi}\left(\mathbf{z}_{t}\right) \\
\mathbf{z}_{k t} & =\mathbf{z}_{k t-1} \oplus \boldsymbol{\eta}_{k t}, \quad \quad \boldsymbol{\eta}_{k t} \sim \mathcal{L}_{m-1}\left(\mathbf{0}, D_{n} \Upsilon_{k} D_{m}^{\prime}\right)
\end{aligned}
$$

where $\boldsymbol{\phi}\left(\mathbf{z}_{t}\right)=\left(\phi_{A_{1 t}}\left(\mathbf{z}_{1 t}\right), \ldots, \phi_{A_{K t}}\left(\mathbf{z}_{K t}\right)\right)$ is a function from $\mathbb{S}^{m-1}$ to $\mathbb{S}^{n-1}$, where the function $\phi_{A}(\mathbf{z})$ has been defined in 2.3.

The representation in corollary 2.3 shows that the model is a conditionally linear model with link function defined by $\phi_{A}$ and a linear local level factor model on the simplex. Also, by extending the $\odot$ product operation to the case of a matrix of real numbers and exploiting the Euclidean vector space structure of $\left(\mathbb{S}^{n}, \oplus, \odot\right)$ allow us to write the transform $\phi_{A}$, for special values of $A$, as a linear matrix operation between simplices of different dimensions as stated in the following remark. In the following we introduce the symbol $\boxplus$ and define the matrix multiplication operation.

Remark 1. Let $\mathbf{z} \in \mathbb{S}^{m-1}$ be a composition, $A$ a $(n \times m)$ real matrix and define the matrix multiplication $A \boxplus \mathbf{z}=C_{n}\left(\prod_{j=1}^{m} z_{j}^{a_{1 j}}, \ldots, \prod_{j=1}^{m} z_{j}^{a_{n-1 j}}\right)$. If $A$ is such that $A \boldsymbol{\iota}_{m}=\mathbf{0}_{n}$ and $a_{i m}=-1, i=1, \ldots, n-1$ and $a_{n, j}=0 j=1, \ldots, m$, the transform defined in proposition 2.3 can be written as $\phi_{A}(\mathbf{z})=A \boxplus \mathbf{z}$.

A simulated example of compositional factor model is given in Fig. 2 by using the De Finetti or ternary diagram (see Cannings and Edwards (1968) 

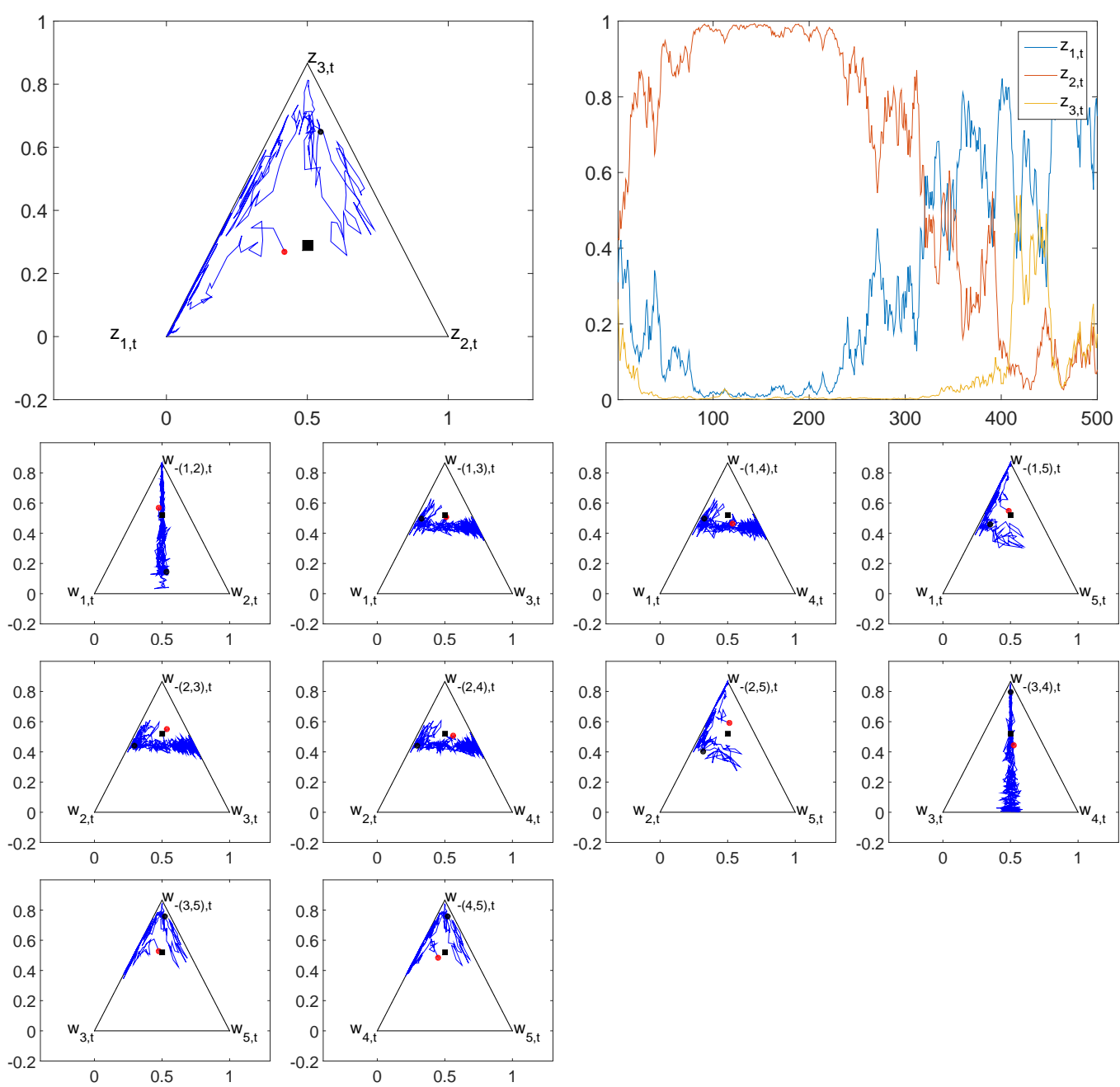

Figure 2: First row: De Finetti's diagram (left) and the time series plot (right) of the ternary $\left(z_{1, t}, z_{2, t}, z_{3, t}\right)$. Other rows: De Finetti's diagram of the ternary $\left(w_{i, t}, w_{j, t}, w_{-(i, j) t}\right), j>i$. In each plot the trajectory (blue line), the starting (red) and ending (black) points and the equal weight composition (square).

and Pawlowsky-Glahn et al. (2015), Supplementary Material S.1). The first row presents the evolution of three driving factors $\left(z_{1, t}, z_{2, t}, z_{3, t}\right)$ by using a De Finetti's diagram (left) and a time series plot (right). The other rows present the pairwise comparisons of the weight dynamics by the De Finetti's diagram of the trajectory (blue line) of the ternary $\left(w_{i, t}, w_{j, t}, w_{-(i, j), t}\right)$ where 
$w_{-(i, j), t}=\sum_{l \neq i, j} w_{l, t}$ is the other model total weight. The red and black dots are the initial and final values. Further details of this example are given in section S.3 of the Supplementary Material. We refer to the Billheimer et al. (2001) for further details on the algebraic structure of the simplex equipped with the perturbation and powering composition and for a Gibbs sampling scheme for compositional state space model. See also Egozcue et al. (2003), Egozcue and Pawlowskky-Glahn (2005) and Fiŝerová and Hron (2011) for further details on the isometric transforms from the real space to the simplex and and for further geometric aspects and property analysis of operations on the simplex, such as the amalgamation and subcomposition operations. See also Pawlowsky-Glahn and Buccianti (2011) and Pawlowsky-Glahn et al. (2015) for up-to-date and complete reviews on compositional data models.

\section{Sequential inference}

The analytic solution of the optimal filtering problem is generally not known, also the clustered-based mapping of the predictor weights onto the subset of latent variables requires the solution of an optimization problem which is not available in closed form. Thus, we apply a sequential numerical approximation of the two problems and use an algorithm which, at time $t$ iterates over the following two steps:

1. Parallel sequential clustering computation of $\Xi_{t}$

2. Sequential Monte Carlo approximation of combination weights and predictive densities 
As regards the sequential clustering, we apply a parallel and sequential kmeans method with a forgetting factor for the sequential learning of the group structure. K-means clustering is a method partitioning a set of $n$ vectors of parameters or features of the predictors, $\boldsymbol{\psi}_{i t}, i=1, \ldots, n$, into $m$ disjoints sets (clusters), in which each observation belongs to the cluster with the least distance. Given a definition of dependence, the k-means will group predictions based on their distance. Moreover, the sequential k-means algorithm is easy to parallelize and it has been done on multi core CPU and GPU computing environments. The details of the algorithm and its parallel implementation are given in the Supplementary Material S.2.

\section{Empirical applications}

As a first example we consider the extended Stock and Watson (2005) dataset, which includes 142 series sampled at a quarterly frequency from 1959Q1 to 2011Q2. Here we focus on obtaining a set of relevant clusters and studying their patterns over time which are valuable signals that may lead to improved macro modeling and forecasting. The second example focuses on replicating the daily Standard \& Poor 500 (S\&P500) index return and predicting the economic value of tail events like Value-at-Risk.

\subsection{A large macroeconomic dataset}

We consider the extended Stock and Watson (2005) dataset, which includes 142 series sampled at a quarterly frequency from 1959Q1 to 2011Q2. A graphical description of the data is given in Figure S.2 in the Supplementary Material. 
The dataset includes only revised series and not vintages of real-time data, when data are revised. See Aastveit et al. (2015) for a real-time application (with a dataset that includes fewer series) of density nowcasting and on the role of model incompleteness over vintages and time. In order to deal with stationary series, we apply the series-specific transformation suggested in Stock and Watson (2005). Let $y_{i t}$ with $i=1, \ldots, n$ and $t=1, \ldots, T$, be the set of transformed variables.

For each variable we estimate a Gaussian autoregressive model of the first order, $\operatorname{AR}(1)$,

$$
y_{i t}=\alpha_{i}+\beta_{i} y_{i t-1}+\zeta_{i t}, \quad \zeta_{i t} \sim \mathcal{N}\left(0, \sigma_{i}^{2}\right)
$$

Then, we identify the clusters of parameters by applying our k-means clustering algorithm on the estimated vectors, $\hat{\boldsymbol{\theta}}_{i}=\left(\hat{\alpha}_{i}, \hat{\beta}_{i}, \hat{\sigma}_{i}^{2}\right)^{\prime}$. Therefore, our predictions are grouped in clusters depending on mean, persistence and volatility properties. Moreover, following the literature on factor models we are interested in the interpretation of the clusters over the full sample and consequently we impose that the cluster allocation of each model is fixed over the forecasting vintages, i.e. $\Xi_{t}=\Xi, t=1, \ldots, T$. See the next section for an example where such an assumption is relaxed. We estimated the model using Bayesian inference and use a normal-inverse-gamma prior with means for $\alpha_{i}$ and the $\beta$ equal to zero and variances equal to 100 . For the variance $\sigma_{i}^{2}$ we use an inverse-gamma with degrees of freedom equal to the number of lags (one) and intercept, that is two. The predictive densities are Student-t distributed and the means (which are the same as the medians in this case) of the densities are used as point forecasts. We assume alternatively 5 and 7 clusters. In the grouping, we identify two clusters related to real activities; one cluster related to prices; and one cluster 
related to financial variables. The other clusters contains the remaining series. A detailed description of the 5 and 7 clusters is provided in Tables S.1-S.2 in the Supplementary Material.

\section{Set-up of the experiment}

We split the sample size 1959Q3-2011Q2 in two periods. The initial 102 observations from 1959Q3-1984Q1 are used as initial in-sample (IS) period; the remaining 106 observations from 1985Q1-2011Q2 are used as an OOS period. The AR models are estimated recursively and $h$-step ahead (Bayesian) $t$-Student predictive densities are constructed using a direct approach extending each vintage with the new available observation; see for example Koop (2003) for the exact formula of the mean, standard deviation and degrees of freedom.

We predict four different series often considered core variables in monetary policy analysis: real GDP growth, inflation measured as price deflator growth, 3-month Treasury Bill rate and total employment. We consider $h=1, \cdots, 5$ step-ahead horizons. For all the variables to be predicted, we apply an AR(1) as benchmark model. Moreover, we also consider the Dynamic Factor Model (DFM) with 5 factors described in Stock and Watson (2012) as another benchmark. This DFM expresses each of the $n$ time series as a component driven by the latent factors plus an idiosyncratic disturbance. More precisely:

$$
\mathbf{y}_{t}=\Lambda \mathbf{f}_{t}+\varepsilon_{t}, \quad \Phi(\mathrm{L}) \mathbf{f}_{t}=\boldsymbol{\eta}_{t}
$$

where the $\mathbf{y}_{t}=\left(y_{1, t}, \ldots, y_{n, t}\right)^{\prime}$ is an $n \times 1$ vector of observed series, $\mathbf{f}_{t}=$ $\left(f_{1, t}, \ldots, f_{r, t}\right)^{\prime}$ is an $r$ vector of latent factors, $\Lambda$ is a $n \times r$ matrix of factors loadings, $\Phi(\mathrm{L})$ is an $r \times r$ matrix lag polynomial, $\boldsymbol{\varepsilon}_{t}$ is an $n$ vector of idiosyncratic 
components and $\boldsymbol{\eta}_{t}$ is an $r$ vector of innovations. In this formulation the term $\Lambda \mathbf{f}_{t}$ is the common component of $\mathbf{y}_{t}$. Bayesian estimation of the model described in equation (20) is carried out using Gibbs Sampling given in Koop and Korobilis (2009).

\begin{tabular}{|c|c|c|c|c|c|c|c|c|c|c|c|c|c|c|c|}
\hline & \multicolumn{3}{|c|}{$\mathrm{h}=1$} & \multicolumn{3}{|c|}{$\mathrm{h}=2$} & \multicolumn{3}{|c|}{$\mathrm{h}=3$} & \multicolumn{3}{|c|}{$\mathrm{h}=4$} & \multicolumn{3}{|c|}{$=5$} \\
\hline & $\mathrm{PE}$ & LS & CRPS & $\mathrm{PE}$ & LS & CRPS & $\mathrm{PE}$ & LS & CRPS & $\mathrm{PE}$ & LS & CRPS & $\mathrm{PE}$ & LS & CRPS \\
\hline & \multicolumn{15}{|c|}{ RGDP } \\
\hline$\overline{\mathrm{AR}}$ & 0.647 & -1.002 & 0.492 & 0.658 & -1.005 & 0.496 & 0.671 & -1.007 & 0.501 & 0.676 & -1.009 & .503 & .682 & 1.009 & .506 \\
\hline BDFM & 0.649 & -1.091 & $0.382^{* *}$ & 0.651 & -1.066 & $0.385^{* * *}$ & 0.654 & -1.138 & $0.388^{* *}$ & 0.652 & -1.060 & $0.384^{* *}$ & 0.655 & -1.099 & $0.388^{* *}$ \\
\hline UDCEW 5 & 50.644 & -0.869 & $0.333^{* *}$ & 0.655 & -0.893 & $0.340^{* * *}$ & $0.657^{*}$ & -0.900 & $0.341^{* *}$ & $0.655^{*}$ & -0.902 & $0.341^{* *}$ & $0.658^{*}$ & -0.912 & $0.343^{* *}$ \\
\hline MDCEW5 & 50.630 & -0.928 & $0.326^{* *}$ & 0.645 & -0.987 & $0.336^{* *}$ & $0.638^{*}$ & -0.924 & $0.330^{* *}$ & $0.637^{*}$ & -0.897 & $0.328^{* *}$ & $0.636^{*}$ & -0.844 & $0.324^{* *}$ \\
\hline UDCLS5 & 0.773 & -1.306 & 0.464 & 0.663 & -1.275 & $0.433^{* *}$ & 0.687 & -1.339 & $0.446^{* *}$ & 0.689 & -1.327 & $0.448^{* *}$ & 0.715 & -1.380 & 0.481 \\
\hline MDCLS5 & 0.725 & -1.145 & 0.505 & $0.591^{*}$ & -1.071 & $0.365^{* *}$ & $0.581^{* * *}$ & -1.041 & $0.340^{* *}$ & $0.591^{*}$ & -1.079 & $0.354^{* * *}$ & $0.557^{*}$ & -1.005 & $.358^{* * *}$ \\
\hline UDC & 0.649 & -0.875 & $0.334^{* *}$ & 0.652 & -0.880 & $0.335^{* *}$ & 0.655 & -0.889 & *** & 0.654 & -0.886 & 0.3 & $0.657^{*}$ & -0.891 & $338^{* *}$ \\
\hline MDCE & 70.642 & -0.979 & $0.334^{* * *}$ & 0.648 & -1.012 & $0.338^{* *}$ & $0.652^{*}$ & -1.016 & $0.342^{*}$ & 0.651 & -1.015 & $0.339^{* *}$ & $0.654^{*}$ & -1.009 & $0.342^{* *}$ \\
\hline UDCLS7 & 0.646 & $-0.868^{*}$ & $0.332^{* * *}$ & 0.645 & -0.905 & $0.338^{* *}$ & $0.650^{*}$ & -0.918 & $0.341^{* *}$ & 0.655 & -0.939 & $0.352^{* * *}$ & $0.657^{*}$ & -0.914 & $0.342^{* *}$ \\
\hline \multirow[t]{2}{*}{ MDCLS7 } & $0.596^{*}$ & $-0.586^{* *}$ & $0.275^{* *}$ & $0.586^{*}$ & $-0.582^{*}$ & $0.275^{* *}$ & 0.6 & -0.632 & 0.288 & $0.588^{*}$ & $-0.637^{*}$ & 0.287 & 0.610 & -0.634 & $0.286^{* *}$ \\
\hline & \multicolumn{15}{|c|}{ GDP deflator } \\
\hline$\overline{\mathrm{AR}}$ & 0.220 & -0.933 & 0.356 & 0.214 & -0.932 & 0.357 & 0.206 & -0.932 & 0.358 & 0.207 & -0.932 & 0.359 & .208 & -0.932 & 0.361 \\
\hline DDE & 0.220 & -0.5 & & & & & & -0.3 & & & & & & & \\
\hline UDC & 0.230 & -0.429 & 0.169 & 0.220 & -0.427 & 0.167 & 0.212 & -0.422 & 0.165 & & -0.425 & & 213 & -0. & 6 \\
\hline MDC & 50.204 & -0.053 & $0.110^{*}$ & 0.205 & -0.285 & 0.115 & & -0.234 & 0.114 & & -0.167 & & 0.204 & -0.194 & 0.113 \\
\hline UDCLS5 & 0.485 & -1.085 & 0.354 & 0.313 & -1.001 & 0.294 & 0.259 & -0.873 & 0.250 & 0.241 & -0.875 & 0.248 & 0.228 & -0.892 & 0.252 \\
\hline & & -0.280 & & & 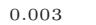 & & & 0.03 & & & & & & & \\
\hline & 0.223 & -0.4 & 0.1 & 0. & -0.42 & & & -0. & 0.163 & & & & & & 4 \\
\hline & 70.208 & $-0.214^{* *}$ & $0.115^{* *}$ & $0.200^{*}$ & $-0.186^{*}$ & 0.11 & $0.197^{*}$ & $-0.172^{* *}$ & $0.109^{* *}$ & 0.197 & $-0.175^{*}$ & $0.110^{*}$ & 0.199 & -0.200 & 0.111 \\
\hline UDCLS7 & 0.235 & -0.50 & $0.179^{* *}$ & 0.220 & -0.5 & 0.18 & 0.224 & -0.514 & 0.1 & 0.221 & -0.516 & 0.1 & 0.214 & -0.475 & 0.171 \\
\hline \multirow[t]{2}{*}{ MDCLS7 } & 0.197 & $0.436^{* *}$ & $0.098^{* *}$ & 0.183 & $0.462^{* *}$ & $0.092^{* *}$ & 0.165 & $0.571^{*}$ & $0.083^{*}$ & 0.160 & $0.570^{* *}$ & $0.082^{*}$ & 0.175 & 0.495 & 0.088 \\
\hline & \multicolumn{15}{|c|}{ th Treasury Bills } \\
\hline$\overline{\mathrm{R}}$ & 0.569 & -1.0 & & 305 & & & 0.518 & -1.038 & 0.343 & 0.5 & & & & & 0. \\
\hline 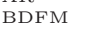 & & -1.1 & & & & & & & & & & & & & \\
\hline & 0.5 & & & & & & & & & & & & & & \\
\hline $\mathrm{MD}$ & $50.517^{* *}$ & $-0.764^{* * *}$ & $0.285^{* *}$ & 0.506 & $-0.752^{* * *}$ & $0.279^{* *}$ & $0.502^{*}$ & $-0.749^{* *}$ & $0.276^{* * *}$ & $0.506^{* *}$ & $-0.755^{* * *}$ & $0.278^{* *}$ & $0.505^{* * *}$ & $-0.751^{* * *}$ & $0.278^{* *}$ \\
\hline & 0.7 & -1.2 & 0.4 & & -1.3 & & & -1.2 & & & -1.2 & & 0.584 & -1.286 & 0.424 \\
\hline & 0.7 & - & & & & & & -1 & & & & & & -1 & \\
\hline & 0.525 & -0.783 & 0.2 & 0.526 & -0.7 & & & -0.7 & $0.284^{*}$ & & & 0.2 & 0.522 & $-0.786^{* *}$ & $0.289^{*}$ \\
\hline & 70.5 & -0.7 & & & & & & & & & & & & & \\
\hline UI & 0.512 & -0.77 & 0.2 & 0.521 & -0.7 & 0.2 & 0.51 & -0.7 & 0.2 & 0.5 & -0. & & 0.5 & -0 & 0.2 \\
\hline \multirow[t]{2}{*}{ MDCLS7 } & 0.488 & -0.72 & 0.270 & 0.484 & $-0.771^{* *}$ & 0.27 & 0.51 & ${ }^{*}-0.755^{* *}$ & 0.283 & 0.513 & -0.771 & 0.283 & 0.496 & -0.73 & 0.275 \\
\hline & \multicolumn{15}{|c|}{ Employment } \\
\hline$\overline{\mathrm{AR}}$ & 0.564 & -1 & 0. & 0 . & -0.999 & 0. & 0 & & 0.4 & & & & & & 0.4 \\
\hline & & & & & & & & & & & & & & & \\
\hline UDCEW5 & $0.585^{*}$ & -0.906 & $0.308^{* *}$ & $0.582^{* *}$ & -0.8 & $0.307^{* *}$ & 0.579 & $-0.955^{* * *}$ & 0.3 & 0.5 & $-0.931^{* *}$ & $0.308^{*}$ & 0.587 & $-0.951^{* *}$ & $0.311^{* *}$ \\
\hline & $50.541^{* *}$ & $-0.926^{* *}$ & 0.277 & $0.554^{* *}$ & $-0.960^{* *}$ & $0.284^{* *}$ & & $-0.917^{* *}$ & 0.2 & & $-0.740^{* *}$ & $0.284^{* *}$ & $0.571^{*}$ & $-0.790^{* *}$ & 0.2 \\
\hline & 0.75 & -1.3 & 0.4 & & -1.2 & & & -1. & & & & & & & \\
\hline & 0.6 & & & & & & & & & & & & & & \\
\hline & 0.535 & -0.801 & & 0.555 & & $0.290^{* *}$ & & $-0.8+2 y-x$ & & 0.577 & $-0.867^{* *}$ & 0.3 & $0.583^{*}$ & $-0.881^{* *}$ & $0.306^{* *}$ \\
\hline & 70.523 & $-0.735^{*}$ & 0.26 & 0.548 & -0.7 & $0.278^{* * *}$ & & -0.8 & & & $-0.855^{* *}$ & 0.2 & $0.578^{*}$ & $-0.885^{* *}$ & $0.297^{* * *}$ \\
\hline & 0.5 & -0 & & & & & & & & & & & & & \\
\hline & 0 & & 0 & 0 & 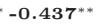 & $0 ?$ & & & & & & & & & \\
\hline
\end{tabular}

Table 1: Forecasting results for $h$ steps ahead. For all the series: root mean square prediction error (PE), logarithmic score (LS) and the continuous rank probability score (CRPS). Bold numbers indicate the best statistic for each horizon and loss function. One or two asterisks indicate that differences in accuracy versus the AR benchmark are statistically different from zero at $5 \%$, and $1 \%$, respectively, using the DieboldMariano $t$-statistic for equal loss. The underlying $p$-values are based on $t$-statistics computed with a serial correlation-robust variance, using the pre-whitened quadratic spectral estimator of Andrews and Monahan (1992).

As we described in Section 2, we consider two alternative strategies for the specification of the parameter matrices $B_{k t}$ : equal weights and score recursive 
weights, where in the second case we fix $g_{i}=L S_{i, h}$ for the various horizons $h$ presented in the following subsection. Further, the predictive densities can be combined with each of the four univariate series and/or with a multivariate approach. As discussed above, we apply two clusters, $k=5$ and 7 . We note that we keep the volatility of the incompleteness term constant. To sum up, we have eight cases defined as UDCEW5 (univariate combination based on 5 clusters with equal weights within clusters), MDCEW5 (multivariate combination based on 5 clusters with equal weights within clusters), UDCLS5 (univariate combination based on 5 clusters with recursive log score weights within clusters), MDCLS5 (multivariate combination based on 5 clusters with recursive log score weights within clusters), UDCEW7 (univariate combination based on 7 clusters with equal weights within clusters), MDCEW7 (multivariate combination based on 7 clusters with equal weights within clusters), UDCLS7 (univariate combination based on 7 clusters with recursive log score weights within clusters), MDCLS7 (multivariate combination based on 7 cluster with recursive log score weights within clusters).

\section{Weight patterns and forecasting results}

Table 1 reports the results to predict real GDP growth, inflation measured as the price deflator of GDP growth, 3-month Treasury Bills and total employment for five different horizons and using three different scoring measures. For all variables, horizons and scoring measures our methodology provides more accurate forecasts than the $\mathrm{AR}(1)$ benchmark and the Bayesian DFM. The Bayesian DFM model provides more accurate forecasts than the $\mathrm{AR}(1)$ for real GDP and inflation at shorter horizons and gives mixed evidence for interest 
rates and employment, but several of our combination schemes outperform this benchmark. The combination that provides the largest gain is the multivariate one based on seven clusters and log score weights within clusters (MCDLS7), resulting in the best statistics 56 times over 60 . In most of the cases, the difference is statistically credible at the $1 \%$ level. This finding extends evidence on the scope for multi-variable forecasting such as in large Bayesian VAR, see e.g. Bańbura et al. (2010) and Koop and Korobilis (2013). Fan charts in Figure S.4 show that the predictions are accurate even at our longest horizon, $h=5$. The variable with low predictive gains is inflation, although our method provides credibly more accurate scores at (at least) $5 \%$ credible level in 8 cases out of 15 , but none in terms of point forecasting. The multivariate combination based on 5 clusters and equal weights yields accurate forecasts, see clusters MCDEW5. We conclude that combining models using multiple clusters with cluster-based weights provides substantial forecast gains in most cases. Additional gains may be obtained by playing with a more detailed cluster grouping and different performance scoring rules for weights associated with models inside a cluster.

From the analysis of the weight time patterns in Figure 3 (see Figure S.7 in the Supplementary Material for weights in the univariate combination), we notice that the weights for the univariate combination are often less volatile than the weights in the multivariate approach. All figures show that the sixth cluster has a large weight, but several other clusters have also large positive weights, namely, clusters 2, 4, and 5 while clusters 1 and 7 do not receive much weight. Apparently, variables such as Exports, Imports and GDP deflator included in the sixth cluster play an important role in forecasting GDP growth, inflation, interest rate and employment, but this role may differ across variables and horizons. Furthermore, 
the De Finetti diagrams in Figure S.6 in the Supplementary Material underline that the relative importance of each cluster at a given horizon is constant over time and the weight composition is far from the equal weight case.

The forecast gains are similar across horizons for the four variables, that is around $10 \%$ relative to the AR benchmark in terms of RMSPE metrics and even larger for the log score and CRPS measures. However, despite these consistent gains over horizons, the logistic-normal weights in Figure 3 differ across horizons. For example, when forecasting GDP growth (panel 1) cluster 4 has a weight around $20 \%$ at horizons 1 and 5, but half of this value at horizon 3, where clusters 2 and 5 have larger weights. The change is even larger for inflation, where cluster 2 has a $20 \%$ weight at horizon 1 and increases to $40-45 \%$ at horizon 5 . The latter case also occurs when there is substantial instability over time. Changes over horizons are less relevant for the other two predicted variables.

Figure S.8 shows a typical output of the model weights $\left(b_{k, i j}\right)$ in the seven clusters. There are large differences across clusters: for clusters 2, 4, 5 and 6 , only a few models have most of the weights; for the other clusters: 1, 3 and 7, similar weights occur across models. This finding associated with the weights in Figure 3 for the clusters $2,4,5$ and 6 indicates that using recursive time-varying $b_{k, i j}$ weights within the clusters increases forecast accuracy for GDP growth relative to using equal weights. Figure S.8 also indicates that the weights within clusters are much more volatile than the cluster common component, indicating that individual model performances change over time even if information in a given clusters is stable.

Evidence is similar for the GDP deflator and employment, but this finding is less clear for bond returns. For this variable, MDCEW5 also predicts accurately. 

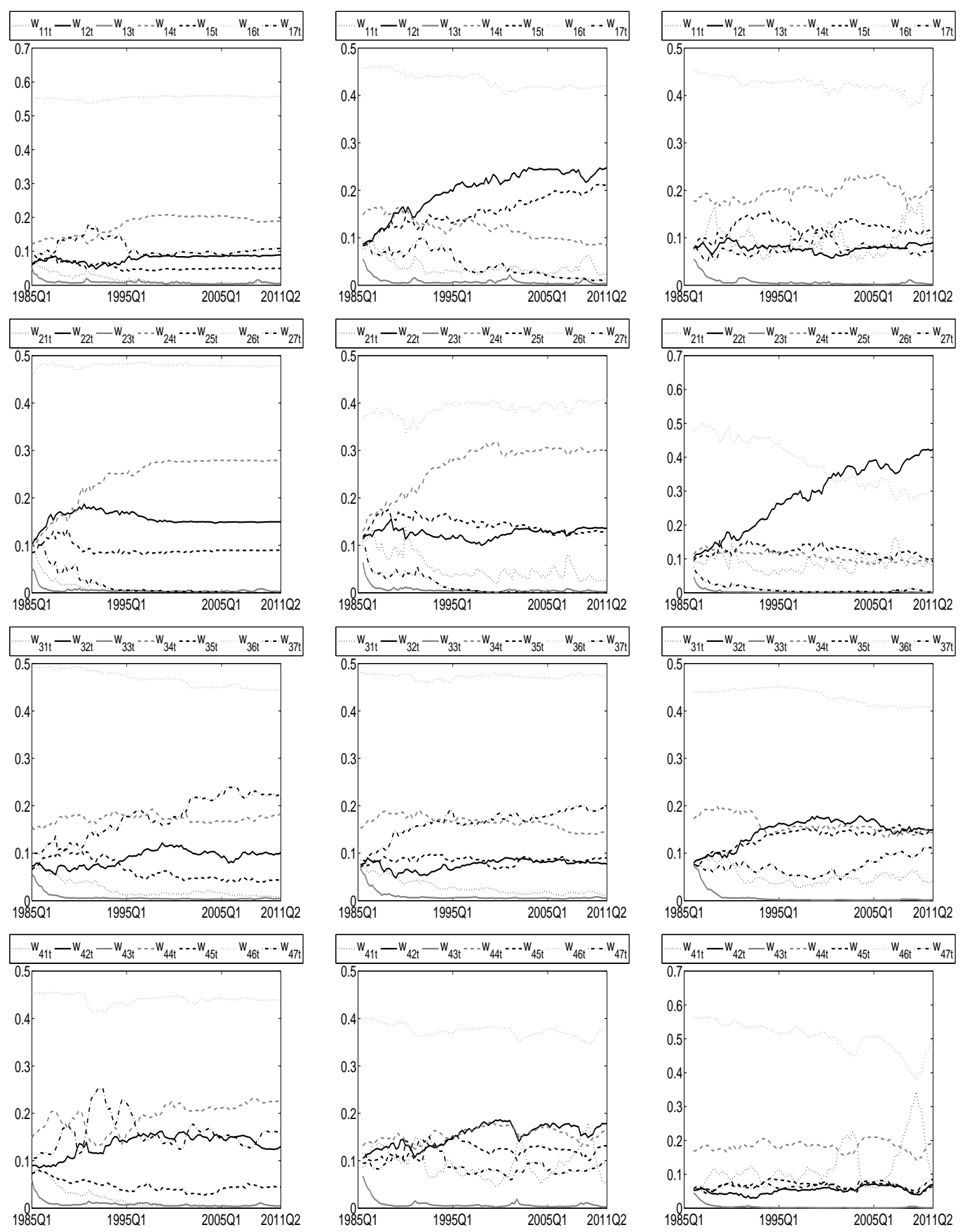

Figure 3: In each plot the logistic-normal weights (different lines) for the multivariate combination model are given. Rows: plot for the four series of interest (real GDP growth rate, GDP deflator, Treasury Bills, employment). Columns: forecast horizons (1, 3 and 5 quarters). 
Also notice that cluster 3, which includes the 3-month Treasury Bills, has the lowest weight in Figures 3. The explanation appears to be that the returns on the 3-month Treasury Bills are modeled with an AR model, which is probably less accurate for the series. Furthermore, the third cluster also contains stock prices and exchange rates that are different from other series with very low persistence and high volatility, making our combination to interpret this cluster more like a noisy component.

We conclude that the logistic-normal weights contain relevant signals about the importance of the forecasting performance of each of the models used in the clusters. Some clusters have a substantial weight while others have only little weight and such a pattern may vary over long time periods. This may lead to the construction of alternative model combinations for more accurate out-of-sample forecasting and it is an interesting line of research to pursue.

\subsection{Predicting Standard \& Poor 500 (S\&P500)}

In this section we report results on replicating the daily Standard \& Poor 500 (S\&P500) index return and predicting the economic value of tail events like Value-at-Risk.

The econometrician interested in predicting this index (or a transformation of it as the return) has, at least, two standard strategies. First, she can model the index with a parametric or non-parametric specification and produce a forecast of it. Second, she can predict the price of each stock $i$ and then aggregate them using an approximation of the unknown weighting scheme.

We propose an alternative strategy based on the fact that many investors, 
including mutual funds, hedge funds and exchange-traded funds, try to replicate the performance of the index by holding a set of stocks, which are not necessarily the exact same stocks included in the index. We collect the S\&P500 index and 3712 individual stock daily prices quoted in the NYSE and NASDAQ from Datastream over the sample March 18, 2002 to December 31, 2009, for a total of 2034 daily observations for each individual series. To control for liquidity we impose that each stock has been traded a number of days corresponding to at least $40 \%$ of the sample size. We compute log returns for all stocks. S\&P500 and cross-section average statistics are reported in Table S.4 in section S.6 of the Supplementary Material. We produce a density forecast for each of the stock prices and then apply our density combination scheme to compute clustered weights and a combined density forecast of the index. The output is a density forecast of the index with clustered weights that indicate the relative forecasting importance of these clusters. That is, a side output of our method is that it produces a replication strategy of the index, providing evidence of which assets track more accurately the aggregate index. We leave a detailed analysis of this last topic for further research.

\section{Individual model estimates}

We estimate a Normal GARCH $(1,1)$ model and a $t$-GARCH $(1,1)$ model via maximum likelihood $(\mathrm{ML})^{1}$ using rolling samples of 1250 trading days (about

\footnotetext{
${ }^{1}$ To ease on the computational workload, we apply the ML approach, which can be seen as approximately Bayes given our large sample
} 
five years) for each stock return:

$$
\begin{aligned}
y_{i t} & =c_{i}+\kappa_{i t} \zeta_{i t} \\
\kappa_{i t}^{2} & =\theta_{i 0}+\theta_{i 1} \zeta_{i, t-1}^{2}+\theta_{2} \kappa_{i, t-1}^{2}
\end{aligned}
$$

where $y_{i t}$ is the log return of stock $i$ at day $t, \zeta_{i t} \sim \mathcal{N}(0,1)$ and $\zeta_{i t} \sim \mathcal{T}\left(\nu_{i}\right)$ for the Normal and t-Student cases, respectively. The number of degrees of freedom $\nu_{i}$ is estimated in the latter model. We produce 784 one day ahead density forecasts from January 1, 2007 to December 31, 2009 using the above equations and the first day ahead forecast refers to January 1, 2007. Our outof-sample (OOS) period is associated with high volatility driven by the US financial crisis and includes, among others, events such as the acquisitions of Bern Stearns, the default of Lehman Brothers and all the following week events. The predictive densities are formed by substituting the ML estimates for the unknown parameters $\left(c_{i}, \theta_{i 0}, \theta_{i 1}, \theta_{i 2}, \nu_{i}\right)$.

As first step, we apply a sequential cluster analysis to our forecasts. We compute two clusters for the Normal GARCH $(1,1)$ model class and two clusters for the $t$-GARCH $(1,1)$ model class. The first two are characterized by low and high volatility density predictions from Normal $\operatorname{GARCH}(1,1)$ models; the third and the fourth ones are characterized by thick or no thick tail density predictions from $t$-GARCH $(1,1)$ models. $^{2}$ The cluster analysis is repeated at every time a new forecast is produced and therefore the cluster composition varies over time. A detailed description of the cluster dynamics is given in section S.6 of the supplementary material.

\footnotetext{
${ }^{2}$ Low degrees of freedom occur jointly with a large scale and high degrees of freedom occur jointly with a low scale.
} 


\section{Weight patterns, model incompleteness and signals of instability}

For convenience, we specified the parameter matrices $B_{k t}$ in equation (8) as equal weights. ${ }^{3}$ We also allow for model incompleteness to be modeled as a timevarying process and estimate $\sigma_{k t}^{2}$ in (2). We label it DCEW-SV and compare it with a combination scheme where $\sigma_{k t}^{2}=\sigma_{k}^{2}$ is time-invariant and label that as DCEW.

Plots of the estimated weights $z_{k, t}$ defined in Corollary 2.1 are shown in Figure 4. The same figure shows the De Finetti's diagrams for a pairwise comparison of the weight dynamics. In the diagrams the blue line represents the trajectory of the ternary $\left(z_{i, t}, z_{j, t}, z_{-(i, j), t}\right)$ where $z_{-(i, j), t}=\sum_{l \neq i, j} z_{l, t}$ is the other model total weight. The red and black dots are the initial and final values.

One can distinguish three different subperiods. In the subperiod before the crisis, the Normal GARCH cluster with high volatility, cluster 2, and the $t$ GARCH cluster with low degrees of freedom, cluster 3, have almost equal high weights while clusters 1 and 4 play a much less important role. In the crisis period of 2008, cluster 3 receives almost all the weight with clusters 1 and 2 almost none. Some of the assets lead the large market decrease in that period. This results in very fat tail densities and our combination scheme takes advantage of this information and assigns to cluster 3 more weight. In the period after the Lehman Brothers collapse cluster 3 receives again a substantial weight while the normal cluster 2, with large variance, is getting gradually more weight. Summarizing, it is seen that the $t$-GARCH$(1,1)$ cluster with small degrees of freedom has most of the period the largest weight. What implications this may have for constructing model combinations that forecast more accurately is a topic for further research.

\footnotetext{
${ }^{3}$ See the macroeconomic case for a comparison with a different scoring rule.
} 

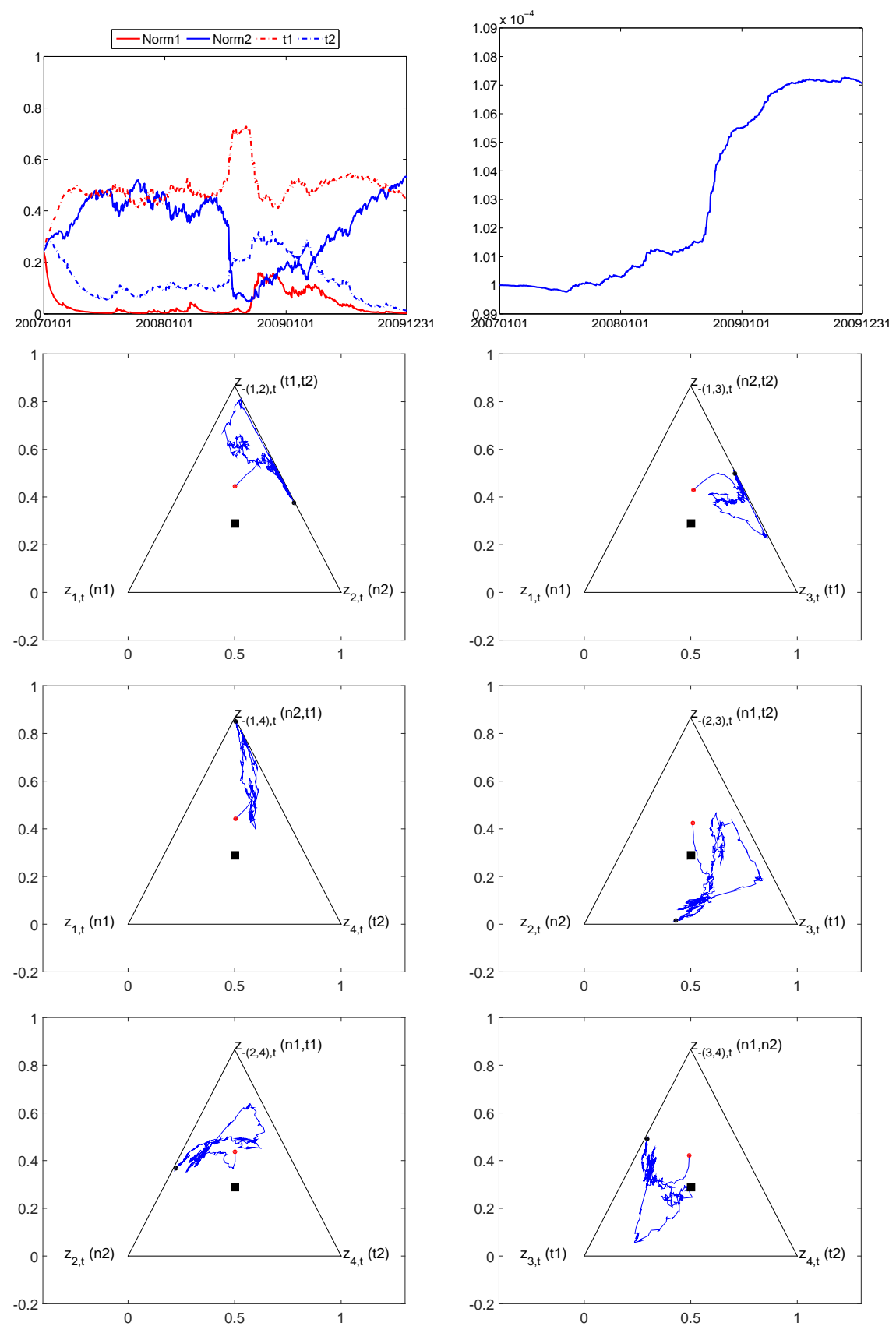

Figure 4: Top left: the mean logistic-normal weights for the two normal GARCH clusters, labeled in the graph "Norm1" and "Norm2", and for the two $t$-GARCH clusters, labeled in the graph " $\mathrm{t} 3$ " and " $\mathrm{t} 4$ ". Top right: posterior mean estimate for $\sigma_{k t}$ in the scheme DCEW-SV. Other rows: De Finetti's diagram for the pairwise subcomposition comparison between model weights over time. In each plot the trajectory of the ternary $\left(z_{i t}, z_{j t}, z_{-(i, j) t}\right), j>i$ (blue line), the starting point (red dot), the ending point (black dot) and the equal weight composition (square). 
Signals of model incompleteness and instability are shown in the top right panel of Figure 4 where plots of the posterior mean estimate for $\sigma_{k t}^{2}$ in the DCEW-SV scheme are presented. The estimates have a $7 \%$ increase in September 2008, which is due to the default of Lehman Brothers and related following events. Interestingly, the volatility does not reduce in 2009, a year with large positive returns opposite the large negative returns in 2008.

From the results so far, we conclude that the combination of several timevarying volatility models with time-varying cluster weights copes with instability in our set of data. There is a clear signal of increased model incompleteness after the 2008 crisis.

\section{Forecast accuracy and economic value}

We compare the performance of our approach to four different benchmarks applied to the S\&P500 log returns: a white noise model (or a random walk for prices), often used as tha main benchmark in equity premium predictability; the Normal GARCH $(1,1)$ and the $t$ - $\operatorname{GARCH}(1,1)$ models described above; and the GJR-GARCH(1,1) model in Glosten et al. (1993) that includes leverage effects. The GJR-GARCH is a richer model than the standard GARCH and should fit the data better. In fact, leverage effect is considered among the stylized facts of financial returns. So the added feature seems relevant in our analysis.

Out-of-sample forecasting result are presented in Table 2. Our combination schemes produce the lowest RMSPE and CRPS and the highest LS. The results indicate that the combination schemes are statistically superior to the no predictability benchmark. The Normal GARCH $(1,1)$ model, the $t$-GARCH $(1,1)$ model and the GJR-GARCH(1,1) model fitted on the index also provide more 
accurate density forecasts than the $\mathrm{WN}$, but not on point forecasting. For all three score criteria, the statistics given by the three individual models are inferior to our combination schemes. Therefore, our strategy that produces forecasts from a large set of assets, clusters them in groups and combines them to predict the S\&P500, produces very accurate point and density forecasts that are superior to no predictability benchmark and classical strategies of modeling directly the index.

Apart from forecasting accuracy, we investigate whether the results documented in the previous paragraphs also possess some economic value. Given that our approach produces complete predictive densities for the variable of interest, it is particularly suitable to compute tail events. We consider two statistics and an economic measure for tail events. We compute weighted averages of Gneiting and Raftery (2007) quantile scores that are based on quantile forecasts that correspond to the predictive densities from the different models, i.e.,

$$
\mathrm{QS}(\alpha, i, t)=\left(\mathrm{I}\left\{y_{t+1} \leqq F^{-1}(\alpha, i)\right\}-\alpha\right)\left(F^{-1}(\alpha, i)-y_{t+1}\right)
$$

with $F^{-1}(\alpha, i)$ is the 1-step ahead quantile forecast using prediction $i$ for level $\alpha \in(0,1)$. It can be shown that integrating $(23)$ over $\alpha \in(0,1)$ will result in the CRPS measure (S.25), see Gneiting and Ranjan (2011). Gneiting and Ranjan (2011), Groen et al. (2013) and Lerch et al. (2016) propose to integrate weighted versions of (23) over $\alpha$, with these weights being fixed functions of $\alpha$ chosen such to emphasize in the forecast evaluation a certain area of the underlying forecast density. We use a discrete approximation to this integration and use weights 
that emphasize both tail and the left tail of the predictive density:

$$
\begin{aligned}
\operatorname{avQS}_{i} & =\frac{1}{T-t_{0}-1} \sum_{s=t_{0}-1}^{T-1}\left(\frac{1}{99} \sum_{j=1}^{99}\left(2 \alpha_{j}-1\right)^{2} \mathrm{QS}\left(\alpha_{j}, i, s+1\right)\right) \\
\operatorname{avQS}_{i, h} & =\frac{1}{T-t_{0}-1} \sum_{s=t_{0}-1}^{T-1}\left(\frac{1}{99} \sum_{j=1}^{99}\left(1-\alpha_{j}\right)^{2} \mathrm{QS}\left(\alpha_{j}, i, s+1\right)\right)
\end{aligned}
$$

where $\alpha_{j}=j / 100$ and $\operatorname{QS}\left(\alpha_{j}, i, s+1\right)$ is defined in (23) for a quantile $j$. In (24), avQS-T emphasizes both tails and avQS-L the left tail of the predictive density relative to the realization 1 -step ahead.

As economic measure, we apply a Value-at-Risk (VaR) based measure, see Jorion (2006). We compare the accuracy of our models in terms of violations, that is the number of times that negative returns exceed the VaR forecast at time $t$, with the implication that actual losses on a portfolio are worse than had been predicted. Higher accuracy results in numbers of violation close to nominal value of $1 \%$. Moreover, to have a gauge of the severity of the violations we compute the total losses by summing the returns over the days of violation for each model.

The last three columns of Table 2 show results for tail evaluation. Our schemes provides the lowest avQS-T and avQS-L statistics, confirming the accuracy of the method in all parts of the distribution. When looking to violations, the number for all individual models is high and above 1\%, with the WN higher than $3 \%$. The dramatic events in our sample, including the Lehman default and all the other features of the US financial crisis, can provide an explanation for the result. However, the two combination schemes provide the best statistics, with violations very close to the $1 \%$ theoretical value. The 
property of our combination schemes to assign higher weights to the fat tail cluster 3 helps to model more accurately the lower tail of the index returns and covers more adequately risks.

Finally, Table S.5 in the supplementary material S.7 compares the execution time of the GPU parallel implementation of our density combination strategy and the CPU multi-core implementation and show large gains from GPU parallelization.

\begin{tabular}{lllllll}
\hline & RMSPE & LS & CRPS & avQS-T & avQS-L & Violation \\
\hline WN & 1.852 & -9.045 & 1.017 & 0.429 & 0.425 & $3.57 \%$ \\
Normal GARCH & 1.852 & $-4.164^{* *}$ & $0.956^{* *}$ & $0.139^{* *}$ & $0.195^{* *}$ & $2.93 \%$ \\
$t$-GARCH & 1.852 & $-2.738^{* *}$ & $0.937^{* *}$ & $0.118^{* *}$ & $0.154^{* *}$ & $2.55 \%$ \\
GJR-GARCH & 1.852 & $-4.068^{* *}$ & $0.955^{* *}$ & $0.125^{* *}$ & $0.158^{* *}$ & $2.75 \%$ \\
DCEW & $\mathbf{1 . 8 1 2}^{* *}$ & $\mathbf{2 . 2 4 9}^{* *}$ & $\mathbf{0 . 9 1 1}^{* *}$ & $\mathbf{0 . 1 1 4}^{* *}$ & $\mathbf{0 . 1 4 9}^{* *}$ & $0.90 \%$ \\
DCEW-SV & $1.816^{* *}$ & $2.206^{* *}$ & $\mathbf{0 . 9 1 3}^{* *}$ & $0.114^{* *}$ & $0.149^{* *}$ & $\mathbf{1 . 0 2} \%$ \\
\hline
\end{tabular}

Table 2: Forecasting results for next day S\&P500 log returns. For all the series are reported the: root mean square prediction error (RMSPE), logarithmic score (LS), the continuous rank probability score (CRPS), tail weighted averages of quantile scores (avQS-T) and left-tail weighted averages of quantile scores (avQS-L). Bold numbers indicate the best statistic for each loss function. One or two asterisks indicate that differences in accuracy from the white noise (WN) benchmark are statistically different from zero at $5 \%$, and 1\%, respectively, using the Diebold-Mariano $t$-statistic for equal loss. The underlying $p$-values are based on $t$-statistics computed with a serial correlation-robust variance, using the pre-whitened quadratic spectral estimator of Andrews and Monahan (1992). The column "Violation" shows the number of times the realized value exceeds the $1 \%$ Value-at-Risk (VaR) predicted by the different models over the sample.

\section{Conclusions}

We proposed in this paper a Bayesian semi-parametric model to construct a time-varying weighted combination of many predictive densities that can deal 
with large data sets in economics and finance. The model is based on clustering the set of predictive densities in mutually exclusive subsets and on a hierarchical specification of the combination weights. This modeling strategy reduces the dimension of the parameter and latent spaces and leads to a more parsimonious combination model. We provide several theoretical properties of the weights and propose the implementation of efficient and fast parallel clustering and sequential combination algorithms.

We applied the methodology to large financial and macro data sets and find substantial gains in point and density forecasting for stock returns and four key macro variables. In the financial applications, we show how 7000 predictive densities based on US individual stocks can be combined to replicate the daily Standard \& Poor 500 (S\&P500) index return and predict the economic value of tail events like Value-at-Risk. In the macroeconomic exercise, we show that combining models for multiple series with cluster-based weights increases forecast accuracy substantially; weights across clusters are very stable over time and horizons, with an important exception for inflation at longer horizons. Furthermore, weights within clusters are very volatile, indicating that individual model performances are very unstable, strengthening the use of density combinations.

The line of research presented in this paper can be extended in several directions. For example, the cluster-based weights contain relevant signals about the importance of the forecasting performance of each of the models used in the these clusters. Some clusters have a substantial weight while others have only little weight and such a pattern may vary over long time periods. This may lead to the construction of alternative model combinations for more accurate out- 
of-sample forecasting and improved policy analysis. We notice also a potential fruitful connection between our approach and research in the field of dynamic portfolio allocation.

\section{References}

Aastveit, K. A., Ravazzolo, F., and van Dijk, H. K. (2015). Combined density nowcasting in an uncertain economic environment. Journal of Business Economics 85 Statistics, forthcoming.

Aitchinson, J. (1982). The statistical analysis of compositional data. Journal of the Royal Statistical Society Series, Series B, 44:139-177.

Aitchinson, J. (1986). The Statistical Analysis of Compositional Data. Chapman \& Hall, London.

Aitchinson, J. (1992). On criteria for measures of compositional difference. Mathematical Geology, 24:365-379.

Aitchinson, J. and Shen, S. M. (1980). Logistic-normal distributions: Some properties and uses. Biometrika, 67:261-272.

Bańbura, M., Giannone, D., and Reichlin, L. (2010). Large Bayesian vector auto regressions. Journal of Applied Econometrics, 25:71-92.

Billheimer, D., Guttorp, P., and Fagan, W. F. (2001). Statistical interpretation of species composition. Journal of the America Statistical Association, 96:12051214.

Billio, M., Casarin, R., Ravazzolo, F., and van Dijk, H. K. (2013). Timevarying combinations of predictive densities using nonlinear filtering. Journal of Econometrics, 177:213-232.

Cannings, C. and Edwards, A. W. F. (1968). Natural selection and the De Finetti diagram. Annals of Human Genetics, 31:421-428. 
Casarin, R., Grassi, S., Ravazzolo, F., and van Dijk, H. K. (2015). Parallel sequential Monte Carlo for efficient density combination: the DeCo Matlab toolbox. Jounal of Statistical Software, 68(3).

Choi, H. and Varian, H. (2012). Predicting the present with Google trends. Economic Record, 88:2-9.

Conflitti, C., De Mol, C., and Giannone, D. (2015). Optimal combination of survey forecasts. International Journal of Forecasting, 31(4):1096-1103.

Del Negro, M., Hasegawa, B. R., and Schorfheide, F. (2015). Dynamic prediction pools: An investigation of financial frictions and forecasting performance. Journal of Econometrics, Forthcoming.

Egozcue, J. J. and Pawlowskky-Glahn, V. (2005). Groups of parts and their balances in compositional data analysis. Mathematical Geology, 37:795-828.

Egozcue, J. J., Pawlowskky-Glahn, V., Mateu-Figueras, G., and Barcelo-Vidal, C. (2003). Isometric logratio transformations for compositional data analysis. Mathematical Geology, 35:279-300.

Ehm, W., Gneiting, T., Jordan, A., and Kruger, F. (2016). Of quantiles and expectiles: consistent scoring functions, choquet representations and forecast rankings. Journal of the Royal Statistical Society: Series B, (78):505-562.

Einav, L. and Levin, J. (2014). Economics in the age of big data. Science, 346(6210):715-718.

Fawcett, N., Kapetanios, G., Mitchell, J., and Price, S. (2015). Generalised density forecast combinations. Journal of Econometrics, 188:150-165.

Fiŝerová, E. and Hron, K. (2011). On the interpretation of orthonormal coordinates for compositional data. Mathematical Geosciences, 43:455-468.

Geweke, J. and Keane, M. (2007). Smoothly mixing regressions. Journal of Econometrics, 138:252-290. 
Glosten, L. R., Jagannathan, R., and Runkle, D. E. (1993). On the relation between the expected value and the volatility of the nominal excess return on stocks. Journal of Finance, 48:1779-1801.

Gneiting, T. and Raftery, A. E. (2007). Strictly proper scoring rules, prediction, and estimation. Journal of the American Statistical Association, 102:359-378.

Gneiting, T. and Ranjan, R. (2011). Comparing density forecasts using threshold and quantile weighted scoring rules. Journal of Business and Economic Statistics, 29:411-422.

Granger, C. W. J. (1998). Extracting information from mega-panels and highfrequency data. Statistica Neerlandica, 52:258-272.

Groen, J. J. J., Paap, R., and Ravazzolo, F. (2013). Real-time inflation forecasting in a changing world. Journal of Business 85 Economic Stastistics, $31: 29-44$.

Grunwald, G. K., Raftery, A. E., and Guttorp, P. (1993). Time series of continuous proportions. Journal of the Royal Statistical Society B, 55:103116.

Jacobs, R. A., Jordan, M. I., Nowlan, S. J., and Hinton, G. E. (1991). Adaptive mixtures of local experts. Journal of Neural Computation, 3:79-87.

Jordan, M. I. and Jacobs, R. A. (1994). Hierarchical mixtures of experts and the EM algorithm. Journal Neural Computation, 6:181-214.

Jordan, M. I. and Xu, L. (1995). Convergence results for the EM approach to mixtures of experts architectures. Neural Networks, 8:1409-1431.

Jorion, P. (2006). Value at Risk: The New Benchmark for Managing Financial Risk. McGraw-Hill, New York.

Koop, G. (2003). Bayesian Econometrics. John Wiley and Sons.

Koop, G. and Korobilis, D. (2009). Bayesian multivariate time series methods for empirical macroeconomics. Foundations and Trends in Econometrics, 3:267358. 
Koop, G. and Korobilis, D. (2013). Large time-varying parameter VARs. Journal of Econometrics, 177:185-198.

Lerch, S., Thorarinsdottir, T., Ravazzolo, R., and Gneiting, T. (2016). Forecaster's dilemma: Extreme events and forecast evaluation. Statistical Science, forthcoming.

Norets, A. (2010). Approximation of conditional densities by smooth mixtures of regressions. Annals of statistics, 38:1733-1766.

Pawlowsky-Glahn, V. and Buccianti, A. (2011). Compositional Data Analysis: Theory and Applications. Wiley.

Pawlowsky-Glahn, V., Egozcue, J. J., and Tolosana-Delgado, R. (2015). Modeling and Analysis of Compositional Data. Wiley.

Peng, F., Jacobs, R. A., and Tanner, M. A. (1996). Bayesian inference in mixtures-of-experts and hierarchical mixtures-of-experts models with an application to speech recognition. Journal of the American Statistical Association, 91:953-960.

Raftery, A. E., Kárńy, M., and Ettler, P. (2010). Online prediction under model uncertainty via Dynamic Model Averaging: Application to a cold rolling mill. Technometrics, 52:52-66.

Stock, J. H. and Watson, W. M. (1999). Forecasting inflation. Journal of Monetary Economics, 44:293-335.

Stock, J. H. and Watson, W. M. (2002). Forecasting using principal components from a large number of predictors. Journal of American Statistical Association, 97:1167-1179.

Stock, J. H. and Watson, W. M. (2005). Implications of dynamic factor models for VAR analysis. Technical report, NBER Working Paper No. 11467.

Stock, J. H. and Watson, W. M. (2012). Disentangling the channels of the 200709 recession. Brookings Papers on Economic Activity, pages 81-156, Spring. 
Stock, J. H. and Watson, W. M. (2014). Estimating turning points using large data sets. Journal of Econometris, 178:368-381.

Varian, H. (2014). Machine learning: New tricks for econometrics. Journal of Economics Perspectives, 28:3-28.

Varian, H. and Scott, S. (2014). Predicting the present with bayesian structural time series. International Journal of Mathematical Modelling and Numerical Optimisation, 5:4-23.

Villani, M., Kohn, R., and Giordani, P. (2009). Regression density estimation using smooth adaptive Gaussian mixtures. Journal of Econometrics, 153:155173.

Waggoner, D. F. and Zha, T. (2012). Confronting model misspecification in macroeconomics. Journal of Econometrics, 171:167-184.

Wood, S. A., Jiang, W., and Tanner, M. (2002). Bayesian mixture of splines for spatially adaptive nonparametric regression. Biometrika, 89:513-528. 


\section{Supplementary Material for "Dynamic Predictive Density Combinations for Large Data Sets in Economics and Finance" by Roberto Casarin, Stefano Grassi, Francesco Ravazzolo and Herman van Dijk}

\section{S.1 Proofs of the results in sections 2 and 3}

Proof of Proposition 2.1 The marginal predictive density is obtained by integrating out the predictors with respect to their distributions. Under regularity condition it is possible to exchange the order of integration and obtain

$$
\begin{aligned}
& f\left(y_{k t} \mid \mathbf{w}_{k t}, \sigma_{k t}^{2}\right) \stackrel{\text { def }}{=} \int_{\mathbb{R}^{n}} f\left(y_{k t} \mid \mathbf{w}_{k t}, \tilde{\boldsymbol{y}}_{t}, \sigma_{k t}^{2}\right) \prod_{j=1}^{n} f_{j t}\left(\tilde{y}_{j t}\right) d \tilde{y}_{j t} \\
& =\sum_{i=1}^{n} w_{i, k t} \int_{\mathbb{R}^{n}} f\left(y_{k t} \mid \tilde{y}_{i t}, \sigma_{k t}^{2}\right) \prod_{j=1}^{n} f_{j t}\left(\tilde{y}_{j t}\right) d \tilde{y}_{j t} \\
& =\sum_{i=1}^{n} w_{i, k t} \int_{\mathbb{R}} f\left(y_{k t} \mid \tilde{y}_{i t}, \sigma_{k t}^{2}\right) f_{i t}\left(\tilde{y}_{i t}\right) d \tilde{y}_{i t}
\end{aligned}
$$

where $f\left(y \mid \mu, \sigma^{2}\right)$ is the pdf of the normal distribution $\mathcal{N}\left(\mu, \sigma^{2}\right)$. Now, by letting $\sigma_{k t}^{2} \rightarrow 0$ for all $k$, one has that $f\left(y_{k t} \mid \mathbf{w}_{k t}, \sigma_{k t}^{2}\right)$ converges to

$$
\sum_{i=1}^{n} w_{k, i t} \int_{\mathbb{R}} \delta_{\tilde{y}_{i t}}\left(y_{k t}\right) f_{i t}\left(\tilde{y}_{i t}\right) d \tilde{y}_{i t}=\sum_{i=1}^{n} w_{i, k t} f_{i t}\left(y_{k t}\right)
$$

$k=1, \ldots, K$.

Proof of Proposition 2.2 See Aitchinson and Shen (1980), Section 2.

Proof of Corollary 2.1 It follows from 2.2 by taking $\mathbf{v}=\mathbf{v}_{k t}$ and $\mathbf{z}=\mathbf{z}_{k t}$.

Proof of Proposition 2.3 It follows from a direct application of the results in Aitchinson and Shen (1980), Section 2.

Proof of Proposition 2.4 From equations 2-6 it is easy to show that the measurement density for each variable of interest is $y_{k t} \sim \mathcal{N}\left(\tilde{\mathbf{y}}_{t}^{\prime} \mathbf{s}_{k t}, \sigma_{k t}^{2}\right)$ with 
$\mathbf{s}_{k t} \sim \mathcal{M}_{n}\left(1, \mathbf{w}_{k t}\right), \quad k=1, \ldots, K$, where $\mathcal{M}_{n}\left(1, \mathbf{w}_{k t}\right)$ denotes a multinomial distribution, and due to the conditional independence assumption one gets the joint measurement density as the product of the variable specific densities.

As regards the transition density, first observe that, thanks to proposition $2.2, \mathbf{z}_{k t}=C_{m}\left(\exp \left(\mathbf{v}_{k t}\right)\right)$ follows $\mathcal{L}_{m-1}\left(D_{m} \mathbf{v}_{k t-1}, D_{m} \Upsilon_{k} D_{m}^{\prime}\right)$. Then note that the multivariate transform $x_{i, k t}=\sum_{j=1}^{m} \xi_{i j, k t} b_{i j, k t} v_{j, k t}, j=1, \ldots, m, i=1, \ldots, n$ implies that $\mathbf{x}_{k t}=A_{k t} \mathbf{v}_{k t}, \mathbf{x}_{k t} \sim \mathcal{N}_{n}\left(A_{k t} \mathbf{v}_{k t-1}, A_{k t} \Upsilon_{k} A_{k t}^{\prime}\right)$, with $A_{k t}=\left(\Xi_{t} \circ B_{k t}\right)$ and that, from Proposition 2.2, $C_{n}\left(A_{k t} \mathbf{v}_{k t}\right)$ follows $\mathcal{L}_{n-1}\left(D_{n} A_{k t} \mathbf{v}_{k t-1}, D_{n} \Upsilon_{k} D_{n}^{\prime}\right)$. Without loss of generality, we assume that $B_{k t}=\boldsymbol{\iota}_{n} \boldsymbol{\iota}_{n}^{\prime}$ and that the $n-\tilde{n}_{t}$ elements in the cluster $m$ correspond to the last $n-\tilde{n}_{t}$ element of $\tilde{\mathbf{y}}_{t}$. This implies the following partition of $\Xi_{t}^{\prime}=\left(\left(\tilde{\Xi}_{t}, O_{\tilde{n}_{t} \times 1}\right)^{\prime},\left(O_{\left(n-\tilde{n}_{t}\right) \times(m-1)}, \boldsymbol{\iota}_{n-\tilde{n}_{t}}\right)^{\prime}\right)$ and of $A_{k t}^{\prime}=\left(\left(\tilde{A}_{k t}, O_{\tilde{n}_{t} \times 1}\right)^{\prime},\left(O_{\left(n-\tilde{n}_{t}\right) \times(m-1)}, \iota_{n-\tilde{n}_{t}}\right)^{\prime}\right)$, where $\left(\tilde{\Xi}_{t}, O_{\tilde{n}_{t} \times 1}\right)$ and $\left(\tilde{A}_{k t}, O_{\tilde{n}_{t} \times 1}\right)$ are a $\left(\tilde{n}_{t} \times m\right)$ matrices. Note that

$$
\begin{aligned}
D_{n} A_{k t} & =\left(I_{n-1},-\iota_{n-1}\right)\left(\left(\tilde{A}_{k t},\left(O_{\tilde{n}_{t} \times 1}\right)^{\prime},\left(O_{\left(n-\tilde{n}_{t}\right) \times(m-1)}, \boldsymbol{\iota}_{n-\tilde{n}_{t}}\right)^{\prime}\right)^{\prime}\right. \\
& =\left(\left(\tilde{A}_{k t},-\boldsymbol{\iota}_{\tilde{n}_{t}}\right)^{\prime}, O_{\left(n-\tilde{n}_{t}-1\right) \times m}^{\prime}\right)^{\prime} \\
& =\left(\tilde{A}_{k t}^{\prime}, O_{\left(n-\tilde{n}_{t}-1\right) \times(m-1)}^{\prime}\right)^{\prime} D_{m}
\end{aligned}
$$

The result then follows by applying Proposition 2.3 to the set of weights $\mathbf{z}_{j, k t}$, $j=1, \ldots, m-1$, with transform coefficients $A=\left(\tilde{A}_{k t}^{\prime}, O_{\left(n-\tilde{n}_{t}\right) \times(m-1)}^{\prime}\right)^{\prime}$.

Proof of Corollary 2.2 The representation follows directly from the application of Proposition 2.2 to $\mathbf{x}_{k t} \sim \mathcal{N}_{n}\left(A_{k t} \mathbf{v}_{k t-1}, A_{k t} \Upsilon_{k} A_{k t}^{\prime}\right)$.

\section{S.2 Sequential approximation of combination weights and predictive densities}

As regards the sequential filtering we apply sequential Monte Carlo as in Billio et al. (2013).

Let $\boldsymbol{\theta}_{t} \in \Theta$ be the parameter vector of the combination model, that is $\boldsymbol{\theta}_{t}=\left(\log \sigma_{1 t}^{2}, \ldots, \log \sigma_{K t}^{2}, \operatorname{vecd}\left(\Upsilon_{1 t}\right), \ldots, \operatorname{vecd}\left(\Upsilon_{K t}\right)\right)$. Let $\mathbf{w}_{t}^{\prime}=\left(\mathbf{w}_{1 t}^{\prime}, \ldots, \mathbf{w}_{k t}\right)$ the vector of weights, and $\mathbf{u}_{1: t}=\left(\mathbf{u}_{1}, \ldots, \mathbf{u}_{t}\right)$ the collection of vectors $\mathbf{u}_{t}$ from time 1 to time $t$. Following Kitagawa (1998), Kitagawa and Sato (2001), and 
Liu and West (2001), we define the augmented state vector $\mathbf{w}_{t}^{\theta}=\left(\mathbf{w}_{t}, \boldsymbol{\theta}_{t}\right) \in \mathcal{Z}$, and the augmented state space $\mathcal{W}=\mathbb{S}^{n-1} \times \Theta$. Our combination model writes in the state space form

$$
\begin{aligned}
\mathbf{y}_{t} & \sim p\left(\mathbf{y}_{t} \mid \mathbf{w}_{t}^{\theta}, \tilde{\mathbf{y}}_{t}\right) & & \text { (measurement density) } \\
\mathbf{w}_{t}^{\theta} & \sim p\left(\mathbf{w}_{t}^{\theta} \mid \mathbf{w}_{t-1}^{\theta}, \mathbf{y}_{1: t-1}, \tilde{\mathbf{y}}_{1: t-1}\right) & & \text { (transition density) } \\
\mathbf{w}_{0}^{\theta} & \sim p\left(\mathbf{w}_{0}^{\theta}\right) & & \text { (initial density) }
\end{aligned}
$$

where the measurement density is

$$
p\left(\mathbf{y}_{t} \mid \mathbf{w}_{t}^{\theta}, \tilde{\mathbf{y}}_{t}\right) \propto \prod_{k=1}^{K} \sum_{i=1}^{n} w_{i, k t} \mathcal{N}\left(\tilde{y}_{i t}, \sigma_{k t}^{2}\right)
$$

and the transition density is the probability density function of the distribution given in equation (14), that is

$$
\begin{aligned}
& p\left(\mathbf{w}_{t} \mid \boldsymbol{\theta}_{t}, \mathbf{w}_{t-1}^{\theta}, \mathbf{y}_{1: t-1}, \tilde{\mathbf{y}}_{1: t-1}\right) \propto \\
& \propto \prod_{k=1}^{K} \delta_{1-\iota_{n-1} \tilde{\mathbf{w}}_{k t}}\left(w_{n, k t}\right)\left(\prod_{j=1}^{n-1} w_{j, k t}\right)^{-1} \prod_{j=1}^{n-1} \exp \left(-\frac{1}{2}\left(\log \left(w_{j, k t} / w_{n, k t}\right)\right.\right. \\
& \left.\left.\left.-\tilde{\tilde{A}}_{k t} D_{m} \boldsymbol{\nu}_{k t-1}\right)\left(\tilde{\tilde{A}}_{k t} D_{m} \Upsilon_{t} D_{m}^{\prime} \tilde{\tilde{A}}_{k t}^{\prime}\right)^{-1}\left(\log \left(w_{j, k t} / w_{n, k t}\right)-\tilde{\tilde{A}}_{k t} D_{m} \boldsymbol{\nu}_{k t-1}\right)^{\prime} \mathrm{S}\right) 10\right)
\end{aligned}
$$

The state predictive and filtering densities are

$$
\begin{aligned}
p\left(\mathbf{w}_{t+1}^{\theta} \mid \mathbf{y}_{1: t}, \tilde{\mathbf{y}}_{1: t}\right) & =\int_{\mathcal{W}} p\left(\mathbf{w}_{t+1}^{\theta} \mid \mathbf{w}_{t}^{\theta}, \mathbf{y}_{1: t}, \tilde{\mathbf{y}}_{1: t}\right) p\left(\mathbf{w}_{t}^{\theta} \mid \mathbf{y}_{1: t}, \tilde{\mathbf{y}}_{1: t}\right) d \mathbf{w}_{t}^{\theta}(\mathrm{S}, 1) \\
p\left(\mathbf{w}_{t+1}^{\theta} \mid \mathbf{y}_{1: t+1}, \tilde{\mathbf{y}}_{1: t+1}\right) & =\frac{p\left(\mathbf{y}_{t+1} \mid \mathbf{w}_{t+1}^{\theta}, \tilde{\mathbf{y}}_{t+1}\right) p\left(\mathbf{w}_{t+1}^{\theta} \mid \mathbf{y}_{1: t}, \tilde{\mathbf{y}}_{1: t}\right)}{p\left(\mathbf{y}_{t+1} \mid \mathbf{y}_{1: t}, \tilde{\mathbf{y}}_{1: t}\right)}
\end{aligned}
$$

The marginal predictive density of the observable variables is

$$
p\left(\mathbf{y}_{t+1} \mid \mathbf{y}_{1: t}\right)=\int_{\mathcal{Y}} p\left(\mathbf{y}_{t+1} \mid \mathbf{y}_{1: t}, \tilde{\mathbf{y}}_{t+1}\right) p\left(\tilde{\mathbf{y}}_{t+1} \mid \mathbf{y}_{1: t}\right) d \tilde{\mathbf{y}}_{t+1}
$$

where $p\left(\mathbf{y}_{t+1} \mid \mathbf{y}_{1: t}, \tilde{\mathbf{y}}_{t+1}\right)$ is defined as

$$
\int_{\mathcal{W} \times \mathcal{Y}^{t}} p\left(\mathbf{y}_{t+1} \mid \mathbf{w}_{t+1}^{\theta}, \tilde{\mathbf{y}}_{t+1}\right) p\left(\mathbf{w}_{t+1}^{\theta} \mid \mathbf{y}_{1: t}, \tilde{\mathbf{y}}_{1: t}\right) p\left(\tilde{\mathbf{y}}_{1: t} \mid \mathbf{y}_{1: t-1}\right) d \mathbf{w}_{t+1}^{\theta} d \tilde{\mathbf{y}}_{1: t}
$$


and represents the conditional predictive density of the observable given the past values of the observable and of the predictors.

\section{S.2.1 Nonlinear sequential filtering}

Each particle set $\Phi_{t}^{j}=\left\{\mathbf{w}_{t}^{\theta i j}, \tilde{\gamma}_{t}^{i j}\right\}_{i=1}^{N}, j=1, \ldots, M$, is updated through the following steps.

1. Conditional combination weights. The approximated state predictive density is

$$
p_{N, j}\left(\mathbf{w}_{t+1}^{\theta} \mid \mathbf{y}_{1: t}, \tilde{\mathbf{y}}_{1: t}^{j}\right)=\sum_{i=1}^{N} p\left(\mathbf{w}_{t+1}^{\theta} \mid \mathbf{w}_{t}^{\theta}, \mathbf{y}_{1: t}, \tilde{\mathbf{y}}_{1: t}^{j}\right) \tilde{\gamma}_{t}^{i j} \delta_{\mathbf{w}_{t}^{\theta i j}}\left(\mathbf{w}_{t}^{\theta}\right)
$$

2. Conditional prediction. The predictive density allows us to obtain the weight predictive density

$$
p_{N, j}\left(\mathbf{z}_{t+1} \mid \mathbf{y}_{1: t+1}, \tilde{\mathbf{y}}_{1: t+1}^{j}\right)=\sum_{i=1}^{N} \gamma_{t+1}^{i j} \delta_{\mathbf{w}_{t+1}^{\theta i j}}\left(\mathbf{w}_{t+1}^{\theta}\right)
$$

where $\gamma_{t+1}^{i j} \propto \tilde{\gamma}_{t}^{i j} p\left(y_{t+1} \mid \mathbf{w}_{t+1}^{\theta i j}, \tilde{\mathbf{y}}_{t+1}^{j}\right)$ is a set of normalized weights, and the observable predictive density

$$
p_{N, j}\left(y_{t+1} \mid \mathbf{y}_{1: t}, \tilde{\mathbf{y}}_{1: t+1}^{j}\right)=\sum_{i=1}^{N} \gamma_{t+1}^{i j} \delta_{y_{t+1}^{i j}}\left(y_{t+1}\right)
$$

where $y_{t+1}^{i j}$ has been simulated from the combination model $p\left(y_{t+1} \mid \mathbf{w}_{t+1}^{\theta i j}, \tilde{\mathbf{y}}_{t+1}^{j}\right)$ independently for $i=1, \ldots, N$.

3. Resampling. Since the systematic resampling of the particles introduces extra Monte Carlo variations and reduces the efficiency of the importance sampling algorithm, we do resampling only when the effective sample size (ESS) is below a given threshold. See Casarin and Marin (2009) for ESS calculation. At the $t+1$ th iteration if $\operatorname{ESS}_{t+1}^{j}<\kappa$, simulate $\Phi_{t+1}^{j}=\left\{\mathbf{w}_{t+1}^{\theta k_{i} j}, \tilde{\gamma}_{t+1}^{i j}\right\}_{i=1}^{N}$ from $\left\{\mathbf{w}_{t+1}^{\theta i j}, \gamma_{t+1}^{i j}\right\}_{i=1}^{N}$ (e.g., multinomial resampling) and set $\tilde{\gamma}_{t+1}^{i j}=1 / N$. We denote with $k_{i}$ the index of the $i$-th re-sampled particle in the original set $\Phi_{t+1}^{j}$. If $\operatorname{ESS}_{t+1}^{j} \geq \kappa$ set $\Phi_{t+1}^{j}=\left\{\mathbf{w}_{t+1}^{\theta i j}, \tilde{\gamma}_{t+1}^{i j}\right\}_{i=1}^{N}$. 


\section{S.2.2 Parallel sequential filtering}

With regard to the filtering part, we use $M$ parallel conditional SMC filters, where each filter is conditioned on the predictor vector sequence $\tilde{\mathbf{y}}_{s}, s=1, \ldots, t$. We initialise independently the $M$ particle sets: $\Phi_{0}^{j}=\left\{\mathbf{w}_{0}^{\theta i j}, \tilde{\gamma}_{0}^{i j}\right\}_{i=1}^{N}, j=$ $1, \ldots, M$. Each particle set $\Phi_{0}^{j}$ contains $N$ i.i.d. random variables $\mathbf{w}_{0}^{\theta i j}$ with random weights $\tilde{\gamma}_{0}^{i j}$. We initialise the set of predictors, by generating i.i.d. samples $\tilde{\mathbf{y}}_{1}^{j}, j=1, \ldots, M$, from $p\left(\tilde{\mathbf{y}}_{1} \mid \mathbf{y}_{0}\right)$ where $\mathbf{y}_{0}$ is an initial set of observations for the variable of interest.

Then, at the iteration $t+1$ of the combination algorithm, we approximate the predictive density $p\left(\tilde{\mathbf{y}}_{t+1} \mid \mathbf{y}_{1: t}\right)$ as follows

$$
p_{M}\left(\tilde{\mathbf{y}}_{t+1} \mid \mathbf{y}_{1: t}\right)=\frac{1}{M} \sum_{j=1}^{M} \delta_{\tilde{\mathbf{y}}_{t+1}^{j}}\left(\tilde{\mathbf{y}}_{t+1}\right)
$$

where $\tilde{\mathbf{y}}_{t+1}^{j}, j=1, \ldots, M$, are i.i.d. samples from the predictive densities and $\delta_{x}(y)$ denotes the Dirac mass at $x$.

We assume an independent sequence of particle sets $\Phi_{t}^{j}=\left\{\mathbf{w}_{1: t}^{\theta i j}, \tilde{\gamma}_{t}^{i j}\right\}_{i=1}^{N}$, $j=1, \ldots, M$, is available at time $t+1$ and that each particle set provides the approximation

$$
p_{N, j}\left(\mathbf{w}_{t}^{\theta} \mid \mathbf{y}_{1: t}, \tilde{\mathbf{y}}_{1: t}^{j}\right)=\sum_{i=1}^{N} \tilde{\gamma}_{t}^{i j} \delta_{\mathbf{w}^{\theta i j}}\left(\mathbf{w}_{t}^{\theta}\right)
$$

of the filtering density, $p\left(\mathbf{w}_{t}^{\theta} \mid \mathbf{y}_{1: t}, \tilde{\mathbf{y}}_{1: t}^{j}\right)$, conditional on the $j$-th predictor realisation, $\tilde{\mathbf{y}}_{1: t}^{j}$. Then $M$ independent conditional SMC algorithms are used to find a new sequence of $M$ particle sets, which include the information available from the new observation and the new predictors. Each SMC algorithm iterates, for $j=1, \ldots, M$, the steps given in section S.2.1.

After collecting the results from the different particle sets, it is possible to obtain the following empirical predictive density

$$
p_{M, N}\left(\mathbf{y}_{t+1} \mid \mathbf{y}_{1: t}\right)=\frac{1}{M N} \sum_{j=1}^{M} \sum_{i=1}^{N} \delta_{\mathbf{y}_{t+1}^{i j}}\left(\mathbf{y}_{t+1}\right)
$$

For horizons $h>1$, we apply a direct forecasting approach (see Marcellino 
et al., 2006) and compute predictive densities $p_{M, N}\left(\mathbf{y}_{t+h} \mid \mathbf{y}_{1: t}\right)$ following the steps previously described.

\section{S.2.3 Sequential Clusering}

The sequential clustering algorithm is summarized as follows. Let $\mathbf{c}_{j 0}, j=$ $1, \ldots, m$, an initial set of random points and let $\mathbf{c}_{j t}, j=1, \ldots, m$ be the centroids, defined as

$$
\mathbf{c}_{j t}=\frac{1}{n_{j t}} \sum_{i \in N_{j t}} \boldsymbol{\psi}_{i t}
$$

where $n_{j t}$ and $N_{j t}$ have been define in the previous sections. At time $t+1$ a new set of observations $\boldsymbol{\psi}_{i t+1} \in \mathbb{R}^{d}, i=1, \ldots, n$ is assigned to the different $m$ groups of observations based on the minimum distance, such as the Euclidean distance, $\|\cdot\|$, between the observations and the centroids $\mathbf{c}_{j t} \in \mathbb{R}^{d}, j=1, \ldots, m$. Assume $j_{i}=\arg \min \left\{j=1, \ldots, m\left|\| \boldsymbol{\psi}_{i t}-\mathbf{c}_{j t}\right| \mid\right\}, i=1, \ldots, n$, then the allocation variable $\xi_{i j, t}$ is equal to 1 if $j=j_{i}$ and 0 otherwise and the centroids are updated as follows

$$
\mathbf{c}_{j t+1}=\mathbf{c}_{j t}+\lambda_{t}\left(\mathbf{m}_{j t+1}-\mathbf{c}_{j t}\right)
$$

where

$$
\mathbf{m}_{j t+1}=\frac{1}{n_{j t+1}} \sum_{i \in N_{j t+1}} \boldsymbol{\psi}_{i t}
$$

and $\lambda_{t} \in[0,1]$. Note that the choice $\lambda_{t}=n_{j t+1} /\left(n_{j t}^{c}+n_{j t+1}\right)$, with $n_{j t}^{c}=\sum_{s=1}^{t} n_{j s}$, implies a sequential clustering with forgetting driven by the processing of the blocks of observations. In the application we fix $\lambda=0.99$.

\section{S.2.4 Parallel sequential clustering}

The parallel implementation of the k-means algorithm can be described as follows, see also Favirar et al. (2008) and the reference therein. Assume, for simplicity, the $n$ data points can be split in $P$ subsets, $N_{p}=\left\{(p-1) n_{p}+\right.$ $\left.1, \ldots, p n_{p}\right\}, p=1 \ldots, P$, with the equal number of elements $n_{P} . \quad P$ is chosen according to the number of available cores.

1. Assign $P$ sets of $n_{P}$ data points to different cores. 
2. For each core $p, p=1, \ldots, P$

2a. find $j_{i}=\arg \min \left\{j=1, \ldots, m \mid\left\|\boldsymbol{\psi}_{i t}-\mathbf{c}_{j t}\right\|\right\}$, for each observation $i \in N_{p}$ assigned to the core $p$.

2.b find the local centroid updates $\mathbf{m}_{p, j t+1}, j=1, \ldots, m$

3. Find the global centroid updates $\mathbf{m}_{j t+1}=1 / P \sum_{p=1}^{P} \mathbf{m}_{p, j t+1}, j=1, \ldots, m$

4. Update the centroids as in Eq. (S.18).

The k-means algorithm is parallel in point 2) and 3) and this can be used in the GPU context as we do in this paper.

\section{S.3 Simulation example}

To provide a graphical illustration of our compositional factor model, a simulated example is presented. Let there be only one variable of interest $y_{1 t}=y_{t}$, with values given by the combination of five predictors (i.e. $K=1$ and $n=5$ )

$$
y_{t}=\sum_{i=1}^{5} \tilde{y}_{i t} s_{i t}+\varepsilon_{t}, \quad \varepsilon_{t} \sim \mathcal{N}(0,0.2)
$$

$t=1, \ldots, T$, where $\tilde{y}_{i t} \sim \mathcal{N}(i, 0.1 i)$ i.i.d. $i=1, \ldots, 5$ are the predictive distributions, $\left(s_{1 t}, \ldots, s_{5 t}\right)^{\prime} \sim \mathcal{M}_{n}\left(1,\left(s_{1 t}, \ldots, s_{5 t}\right)\right)$, and

$$
\left(\begin{array}{l}
w_{1 t} \\
w_{2 t} \\
w_{3 t} \\
w_{4 t} \\
w_{5 t}
\end{array}\right)=\left(\begin{array}{rrr}
1 & 0 & -1 \\
1 & 0 & -1 \\
0 & 1 & -1 \\
0 & 1 & -1 \\
0 & 0 & 0
\end{array}\right) \odot\left(\begin{array}{l}
z_{1 t} \\
z_{2 t} \\
z_{3 t}
\end{array}\right) \oplus\left(\begin{array}{l}
\varsigma_{1 t} \\
\varsigma_{2 t} \\
\varsigma_{3 t} \\
\varsigma_{4 t} \\
\varsigma_{5 t}
\end{array}\right)
$$

with $\left(\varsigma_{1 t}, \varsigma_{2 t}, \varsigma_{3 t}, \varsigma_{4 t}\right)^{\prime} \sim \mathcal{L}_{4}\left(\mathbf{0}_{4}, 0.1 D_{5} D_{5}^{\prime}\right)$ i.i.d. and $\varsigma_{5 t}=1-\varsigma_{1 t}-\ldots-\varsigma_{4 t}$.

For expository purposes, in order to show graphically the relationship between the components of $\mathbf{w}_{t}$, which are on the 4-dimension simplex, we assume $\mathbf{w}_{t}$ is a transform of $\mathbf{z}_{t}$ with some noise. The dynamics of the latent factors on 
the simplex of dimension 2 are given by

$$
\left(\begin{array}{l}
z_{1 t} \\
z_{2 t} \\
z_{3 t}
\end{array}\right)=\frac{1}{3}\left(\begin{array}{c}
1 \\
1 \\
1
\end{array}\right) \oplus\left(\begin{array}{l}
z_{1 t-1} \\
z_{2 t-1} \\
z_{3 t-1}
\end{array}\right) \oplus\left(\begin{array}{l}
\eta_{1 t} \\
\eta_{2 t} \\
\eta_{3 t}
\end{array}\right)
$$

with $\left(\eta_{1 t}, \eta_{2 t}\right) \sim \mathcal{L}_{2}\left(\mathbf{0}_{2}, 0.2 D_{3} D_{3}^{\prime}\right)$ i.i.d. and $\eta_{3 t}=1-\eta_{1 t}-\eta_{2 t}$. We generate a trajectory of $T=500$ points from the latent factor process (blue line in the top-left chart of Fig. S.1) starting at $\mathbf{z}_{0}=\boldsymbol{\iota}_{3} / 3$ (black dot). The top-right chart of the same figure shows the scatter plot of $w_{k t}, k=2,3,4$ against the first weight $w_{1 t}$. One can easily see that $w_{2 t}$ moves along the same direction of $w_{1 t}$, that is it lies on the 45-degree line, whereas $w_{3 t}$ and $w_{4 t}$ move together and their relationship with $w_{1 t}$ reflects the relationship between $z_{1}$ and $z_{2 t}$. The bottom-left chart shows the trajectory of $y_{t}$ which exhibits a change in mean and variance following the features of the largest combination weight at time $t$ (see bottom-right chart).

\section{S.4 Forecast evaluation}

To shed light on the predictive ability of our methodology, we consider several evaluation statistics for point and density forecasts previously proposed in the literature. Suppose we have $i=1, \ldots, n$ different approaches to predict the variable $y$. We compare point forecasts in terms of Root Mean Square Prediction Errors (RMSPE)

$$
R M S P E_{i, h}=\sqrt{\frac{1}{t^{*}} \sum_{t=\underline{t}}^{\bar{t}} e_{i, t+h}}
$$

where $t^{*}=\bar{t}-\underline{t}+h, \bar{t}$ and $\underline{t}$ denote the beginning and end of the evaluation period, and $e_{i, t+h}$ is the $h$-step ahead square prediction error of model $i$.

The complete predictive densities are evaluated using the Kullback Leibler Information Criterion (KLIC)-based measure, utilising the expected difference in the Logarithmic Scores of the candidate forecast densities; see, for example, Mitchell and Hall (2005), Hall and Mitchell (2007), Amisano and Giacomini (2007), Kascha and Ravazzolo (2010), Billio et al. (2013), and Aastveit et al. 

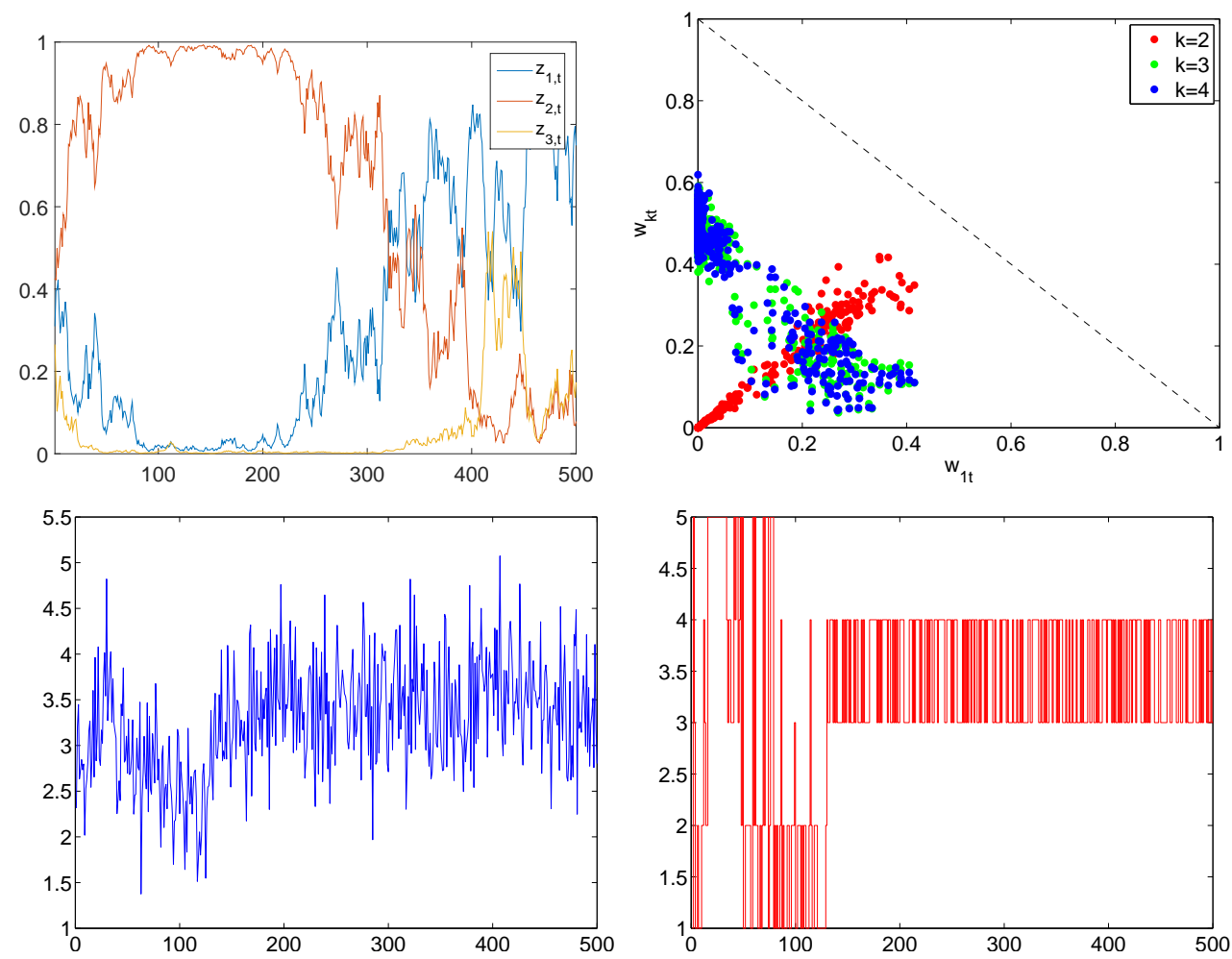

Figure S.1: Simplicial random walk trajectory $\mathbf{z}_{t}$ (top-left), scatter plot of elements of the latent weight vector $\mathbf{w}_{t}$ (top-right), observable process $y_{t}$ (bottom-left) and the largest weight indicator $w_{t}^{*}=\max \left\{w_{k t}, k=1, \ldots, 5\right\}$ (bottom-right).

(2014).

The KLIC is the distance between the true density $p\left(y_{t+h} \mid \mathbf{y}_{1: t}\right)$ of a random variable $y_{t+h}$ and some candidate density $p_{i}\left(y_{t+h} \mid \mathbf{y}_{1: t}\right)$ obtained from the approach $i$ and chooses the model that on average gives the higher probability to events that actually occurred. An estimate of it can be obtained from the average of the sample information, $y_{\underline{t}+1}, \ldots, y_{\bar{t}+1}$, on $p\left(y_{t+h} \mid \mathbf{y}_{1: t}\right)$ and $p_{i}\left(y_{t+h} \mid \mathbf{y}_{1: t}\right)$ :

$$
\overline{K L I C}_{i, h}=\frac{1}{t^{*}} \sum_{t=\underline{t}}^{\bar{t}}\left[\ln p\left(y_{t+h} \mid \mathbf{y}_{1: t}\right)-\ln p_{i}\left(y_{t+h} \mid \mathbf{y}_{1: t}\right)\right]
$$

Although we do not know the true density, we can still compare different densities, $p_{i}\left(y_{t+h} \mid \mathbf{y}_{1: t}\right), i=1, \ldots, n$. For the comparison of two competing models, it is sufficient to consider the Logarithmic Score (LS), which corresponds 
to the latter term in the above sum,

$$
L S_{i, h}=-\frac{1}{t^{*}} \sum_{t=\underline{t}}^{\bar{t}} \ln p_{i}\left(y_{t+h} \mid \mathbf{y}_{1: t}\right)
$$

for all $i$ and to choose the model for which it is minimal, or, as we report in our tables and use in the learning strategies, its opposite is maximal.

Secondly, we also evaluate density forecasts based on the continuous rank probability score (CRPS); see, for example, Gneiting and Raftery (2007), Gneiting and Ranjan (2013), Groen et al. (2013) and Ravazzolo and Vahey (2014). The CRPS for the model $i$ measures the average absolute distance between the empirical cumulative distribution function (CDF) of $y_{t+h}$, which is simply a step function in $y_{t+h}$, and the empirical CDF that is associated with model $i$ 's predictive density:

$$
\begin{aligned}
\operatorname{CRPS}_{i, t+h} & =\int_{-\infty}^{+\infty}\left(F(z)-\mathbb{I}_{\left[y_{t+h},+\infty\right)}(z)\right)^{2} \mathrm{~d} z \\
& =\mathbb{E}_{t}\left|\tilde{y}_{i, t+h}-y_{t+h}\right|-\frac{1}{2} \mathbb{E}_{t}\left|\tilde{y}_{i, t+h}^{*}-\tilde{y}_{i, t+h}^{\prime}\right|
\end{aligned}
$$

where $F$ is the CDF from the predictive density $p_{i}\left(y_{t+h} \mid \mathbf{y}_{1: t}\right)$ of model $i$ and $\tilde{y}_{i, t+h}^{*}$ and $\tilde{y}_{i, t+h}^{\prime}$ are independent random variables with common sampling density equal to the posterior predictive density $p_{i}\left(y_{t+h} \mid \mathbf{y}_{1: t}\right)$. We report the sample average CRPS:

$$
\operatorname{CRPS}_{i, h}=-\frac{1}{t^{*}} \sum_{t=\underline{t}}^{\bar{t}} \operatorname{CRPS}_{i, t+h}
$$

Smaller CRPS values imply higher precisions and, as for the log score, we report the average $\mathrm{CRPS}_{i, h}$ for each model $i$ in all tables.

Finally, following Clark and Ravazzolo (2015), we apply the Diebold and Mariano (1995) t-tests for equality of the average loss (with loss defined as squared error, log score, or CRPS). In our tables presented below, differences in accuracy that are statistically different from zero are denoted by one, two, or three asterisks, corresponding to significance levels of $10 \%, 5 \%$, and $1 \%$, respectively. The underlying $p$-values are based on $t$-statistics computed with 
a serial correlation-robust variance, using the pre-whitened quadratic spectral estimator of Andrews and Monahan (1992). Monte Carlo evidence in Clark and McCracken (2015) and Clark and McCracken (2011) indicates that, with nested models, the Diebold-Mariano test compared against normal critical values can be viewed as a somewhat conservative (conservative in the sense of tending to have size modestly below nominal size) test for equal accuracy in the finite sample. Since the AR benchmark is always one of the model in the combination schemes, we treat each combination as nesting the baseline, and we report $p$-values based on one-sided tests, taking the AR as the null and the combination scheme in question as the alternative.

\section{S.5 Additional details on the macroeconomic dataset}

We consider the extended Stock and Watson (2005) dataset, which includes 142 series sampled at a quarterly frequency from 1959Q1 to 2011Q2. A graphical description of the data is given in Figure S.2.

For each variable we estimate a Gaussian autoregressive model of the first order, $\operatorname{AR}(1)$,

$$
y_{i t}=\alpha_{i}+\beta_{i} y_{i t-1}+\zeta_{i t}, \quad \zeta_{i t} \sim \mathcal{N}\left(0, \sigma_{i}^{2}\right)
$$

using the first 60 observations from each series. Then we identify the clusters of parameters by applying our k-means clustering algorithm on the vectors, $\hat{\boldsymbol{\theta}}_{i}=\left(\hat{\alpha}_{i}, \hat{\beta}_{i}, \hat{\sigma}_{i}^{2}\right)^{\prime}$, of least square estimates of the $\mathrm{AR}(1)$ parameters. A detailed description of the 5 and 7 clusters is provided in Tables S.1-S.2.

The left and right columns in Fig. S.3 show the clusters of series in the parameter space. The results show substantial evidence of different time series characteristics in several groups of series. The groups are not well separated when looking at the intercept values (see Fig. S.3, first and second row). However, the groups are well separated along two directions of the parameter space, which are the one associated with the variance and the one associated with persistence parameters (Fig. S.3, last row). The differences in terms of persistence, in the different groups, is also evident from the heat maps given in Fig. S.5. Different gray levels in the two graphs show the value of the variables (horizontal axis) over time (vertical axis). The vertical red lines indicate the different clusters. 
Table S.1: Predictors classification in 5 clusters (columns).

\begin{tabular}{|c|c|c|c|c|}
\hline 1 & 2 & 3 & 4 & 5 \\
\hline NAPMprodn & Exports & RGDP & Cons-Dur & Cons-Serv \\
\hline CapacityUtil & PGDP & Cons & Imports & FixedInv \\
\hline Emptotal & PCED & Cons-NonDur & GovFed & NonResInv \\
\hline Empgdsprod & CPI-ALL & GPDInv & IPfuels & NonResInv-Struct \\
\hline Empdblegds & PCED-Core & Gov & Ul5wks & NonResInv-Bequip \\
\hline Empservices & CPI-Core & GovStateLoc & U5-14wks & Res.Inv \\
\hline EmpTTU & PCED-DUR-HHEQ & IPconsgds & Orders(NDCapGoods) & IPtotal \\
\hline Empwholesale & PCED-DUR-OTH & IPconsdble & PCED-DUR & IPproducts \\
\hline EmpFIRE & PCED-NDUR & IP:consnondble & PCED-DUR-MOTOR & IPfinalprod \\
\hline Avghrs & PCED-NDUR-FOOD & Empmining & PCED-NDUR-OTH & IP:buseqpt \\
\hline HStartsTotal & PCED-NDUR-CLTH & EmpCPStotal & PFI-NRES & IPmatls \\
\hline BuildPermits & PCED-NDUR-ENERGY & Overtimemfg & PFI-NRES-EQP & IPdblemats \\
\hline HStartsNE & PCED-SERV & Umeanduration & Pimp & IP:nondblemats \\
\hline HStartsMW & PCED-SERV-HOUS & U15-26wks & LaborProd & IPmfg \\
\hline HStartsSouth & PCED-SERV-HOUSOP & Orders(ConsGoods) & RealCompHour & Empconst \\
\hline HStartsWest & PCED-SERV-H0-ELGAS & Comspotprice(real) & 3moT-bill & Empmfg \\
\hline PMI & PCED-SERV-HO-OTH & OilPrice(Real) & 6moT-bill & Empnondbles \\
\hline NAPMnewordrs & PCED-SERV-TRAN & RealAHEgoods & 5yrT-bond & Empretail \\
\hline NAPMvendordel & PCED-SERV-MED & RealAHEmfg & 10yrT-bond & EmpGovt \\
\hline NAPMInvent & PCED-SERV-REC & UnitLaborCost & Reservesnonbor & Helpwantedindx \\
\hline NAPMcomprice & PCED-SERV-OTH & Aaabond & ExrateSwitz & Helpwantedemp \\
\hline Consumerexpect & PGPDI & Baabond & ExrateJapan & EmpCPSnonag \\
\hline fygm10-fygm3 & PFI & Exrateavg & DJIA & EmpHours \\
\hline Fyaaac-fygt10 & PFI-NRES-STRPrInd & ExrateUK & & Uall \\
\hline \multirow[t]{13}{*}{ Fyaaac-fygt10 } & PFI-RES & EXrateCanada & & U15pwks \\
\hline & Pexp & S\&P500 & & U27pwks \\
\hline & Pgov & S\&Pindust & & RealAHEconst \\
\hline & PgovFed & S\&Pdivyield & & Conscredit \\
\hline & Pgovstatloc & S\&PPEratio & & fygm1-fygm3 \\
\hline & FedFunds & fygm6-fygm3 & & \\
\hline & 1yrT-bond & & & \\
\hline & M1 & & & \\
\hline & MZM & & & \\
\hline & M2 & & & \\
\hline & MB & & & \\
\hline & Reservestot & & & \\
\hline & BUSLOANS & & & \\
\hline
\end{tabular}


Table S.2: Predictors classification in 7 clusters (columns).

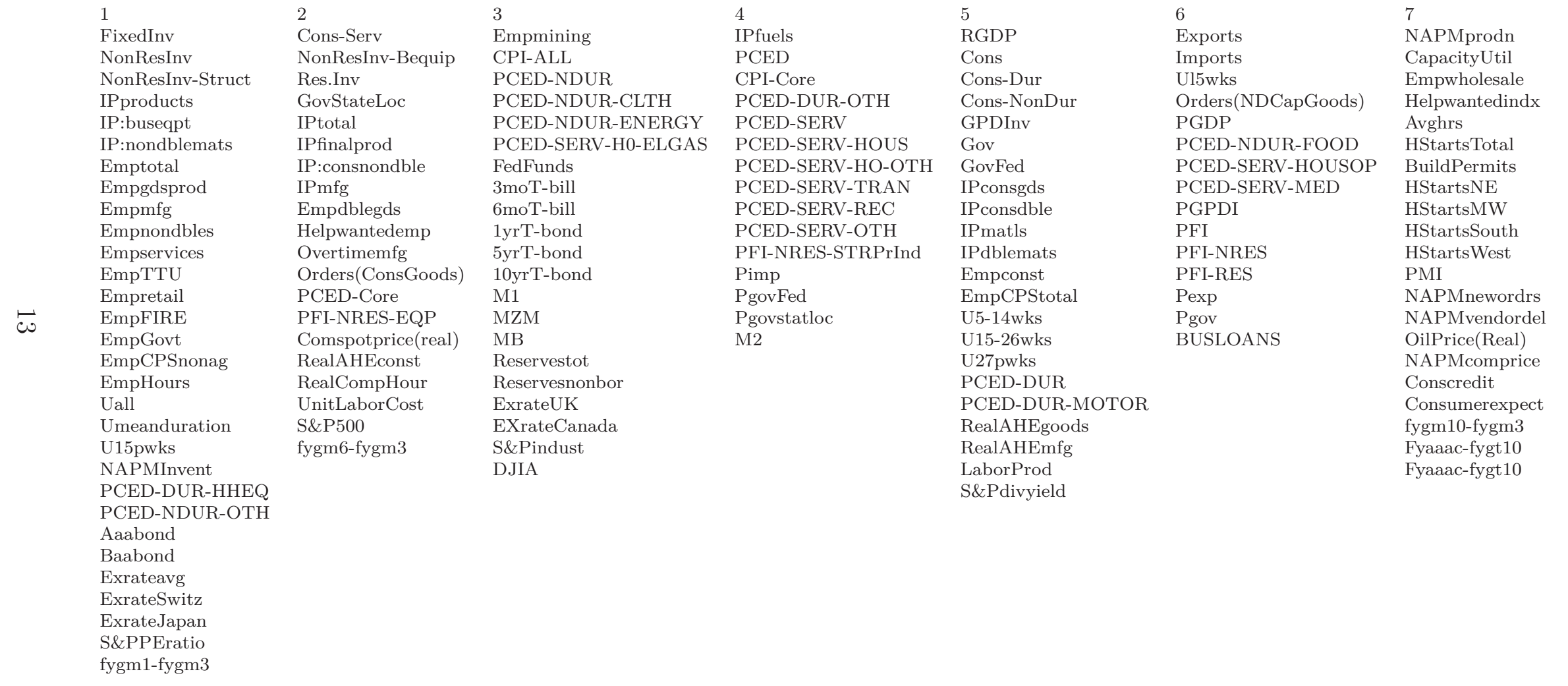




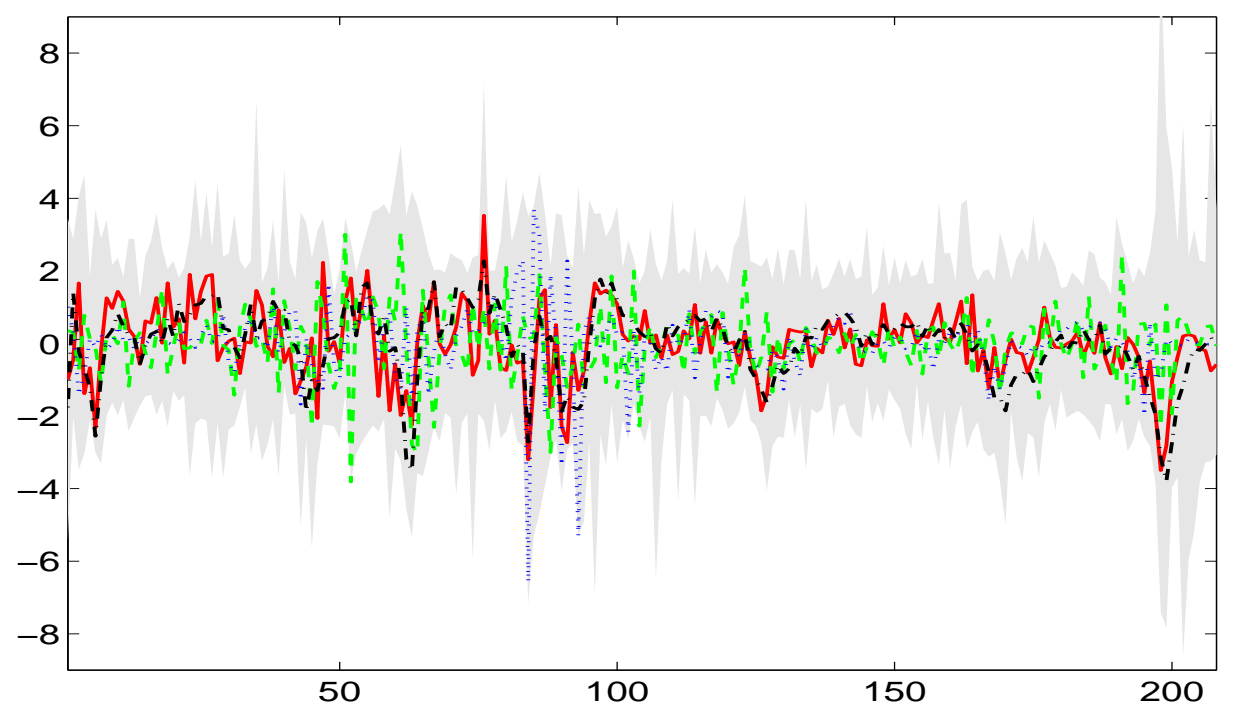

Figure S.2: Gray area: the set of series (standardised for a better graphical representation), at the monthly frequency, of the Stock and Watson dataset. Solid line: growth rate of real GDP (seasonally adjusted) for the US. Dashed line: inflation measured as the change in the GDP deflator index (seasonally adjusted). Dotted line: yields on US government 90-day T-Bills (secondary market). Dashed-dotted: total employment growth rate for private industries (seasonally adjusted).

One can see for example that the series in the 2 nd and 4 th cluster (of 5) are more persistent then the series in the clusters 1, 3 and 5 (see also Fig. S.3, bottom left). Series in cluster 1,2 and 4 are less volatile than series in the cluster 3 and 5 . This information is also summarised by the mean value of the parameter estimates for the series that belong to the same cluster. See the values in Table S.3. Looking at the composition of the predictor groups (see also Tables S.1-S.2), we find for the five clusters that:

1. The first cluster comprises capacity utilisation, employment variables, housing (building permits and new ownership started) and manufacturing variables (new orders, supplier deliveries index, inventories).

2. The second cluster contains exports, a large numbers of price indexes (e.g. prices indexes for personal consumption expenditures, and for gross domestic product) some money market variables (e.g. M1 and M2).

3. The third cluster includes real gross domestic product, consumption and 


\begin{tabular}{|c|ccc|}
\hline \multicolumn{4}{|c|}{5 clusters } \\
\hline$k$ & $\alpha$ & $\beta$ & $\sigma^{2}$ \\
\hline 1 & 0.049 & 0.752 & 0.270 \\
2 & 0.021 & -0.074 & 0.390 \\
3 & 0.124 & 0.157 & 1.260 \\
4 & 0.054 & -0.338 & 1.335 \\
5 & 0.100 & 0.466 & 0.811 \\
\hline
\end{tabular}

\begin{tabular}{|c|ccc|}
\hline \multicolumn{4}{|c|}{7 clusters } \\
\hline$k$ & $\alpha$ & $\beta$ & $\sigma^{2}$ \\
\hline 1 & 0.109 & 0.434 & 0.454 \\
2 & 0.185 & 0.263 & 0.862 \\
3 & 0.019 & -0.116 & 0.224 \\
4 & 0.090 & -0.321 & 0.665 \\
5 & 0.137 & 0.091 & 1.250 \\
6 & 0.124 & -0.437 & 1.297 \\
7 & 0.026 & 0.817 & 0.197 \\
\hline
\end{tabular}

Table S.3: Cluster means for the 5 (top table) and 7 (bottom table) cluster analysis. The first column, $k$, indicates the cluster number given in Fig. S.3 and the remaining three columns the cluster mean along the different directions of the parameter space.

consumption of non-durables, some industrial production indexes, and some financial market variables (e.g., S\&P industrial, corporate bonds and USD - GBP exchange rate).

4. The fourth cluster includes imports, some price indexes and financials such as government debt (3- and 6-months T-bills and 5- and 10-years T-bonds), stocks and exchange rates.

5. The fifth cluster mainly includes investments, industrial production indexes (total and many sector indexes), and employment.

Evidence is similar for the seven clusters. 

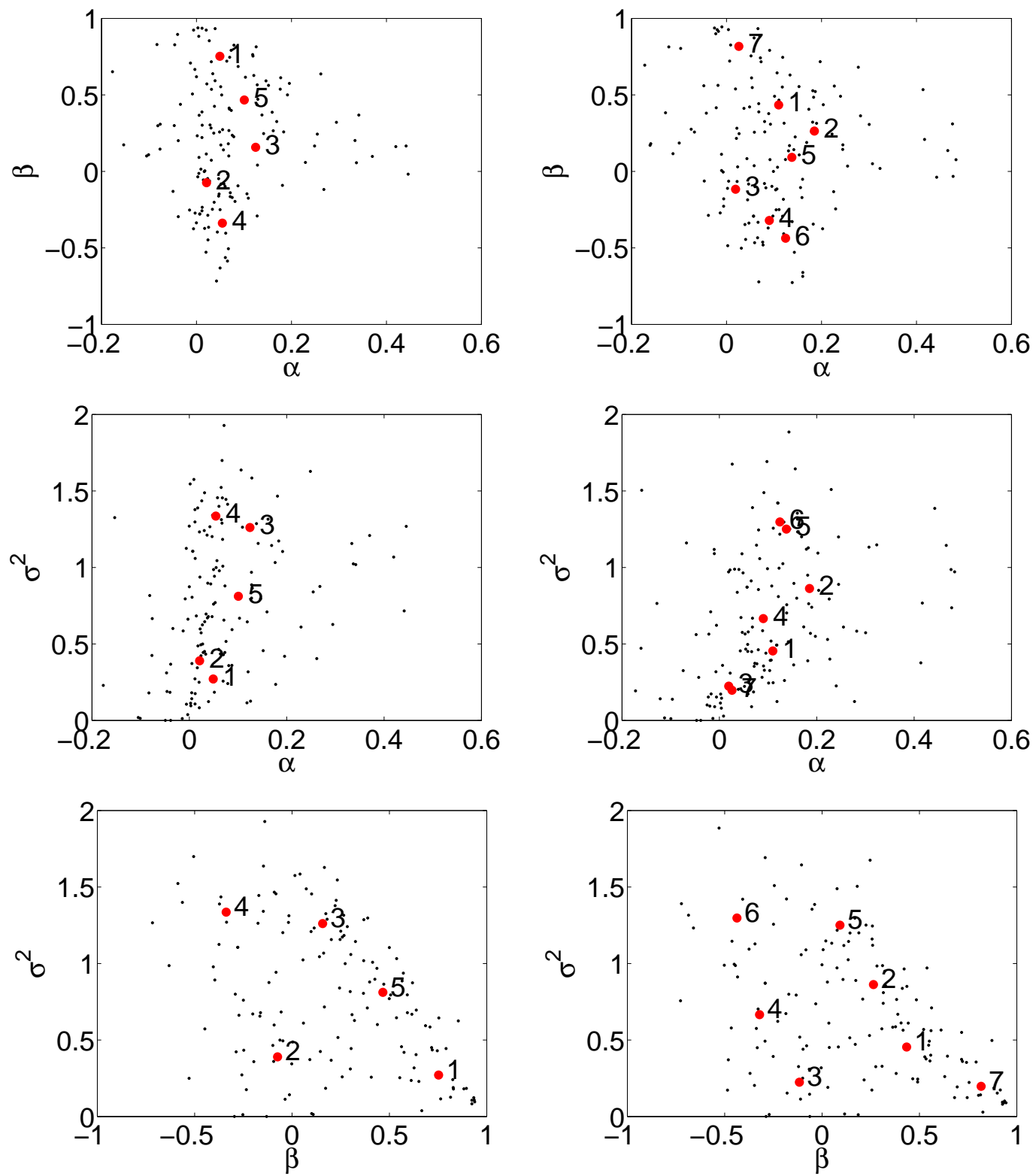

Figure S.3: Pairwise scatter plots of the series features: $\alpha_{i}$ and $\beta_{i}$ (first row), $\alpha_{i}$ and $\sigma_{i}^{2}$ (second row) and $\beta_{i}$ and $\sigma_{i}^{2}$ (last row). In each plot the red dots represent the cluster means. We assume alternatively 5 (left) and 7 (right) clusters. 

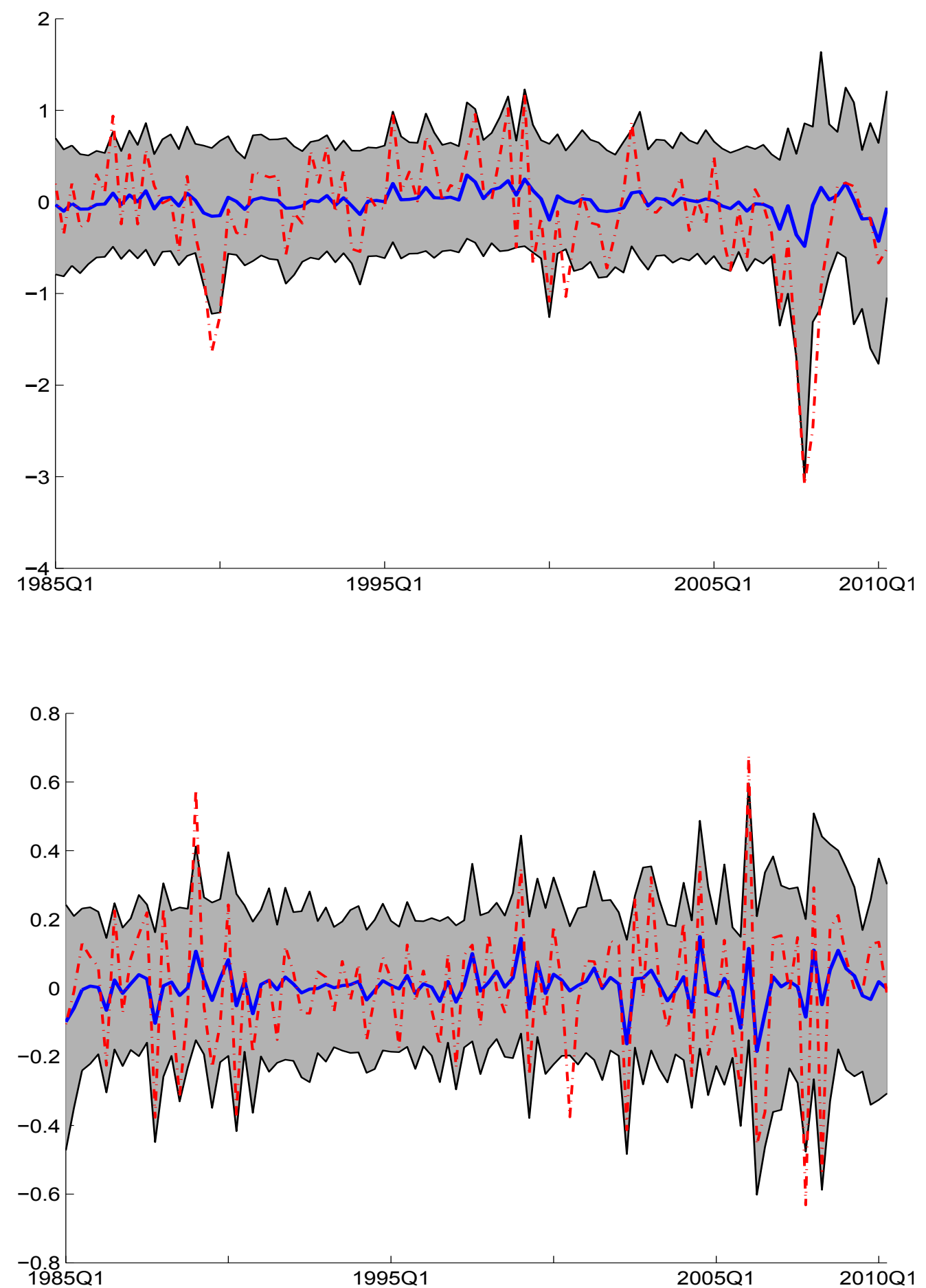

Figure S.4: 5-step ahead fan charts for demeaned GDP (top panel) and demeaned GDP deflator (bottom panel). Estimated mean (solid blue line) and 5\% and 95\% quantiles (gray area) of the marginal prediction density. (Demeaned) realizations in red dashed line 

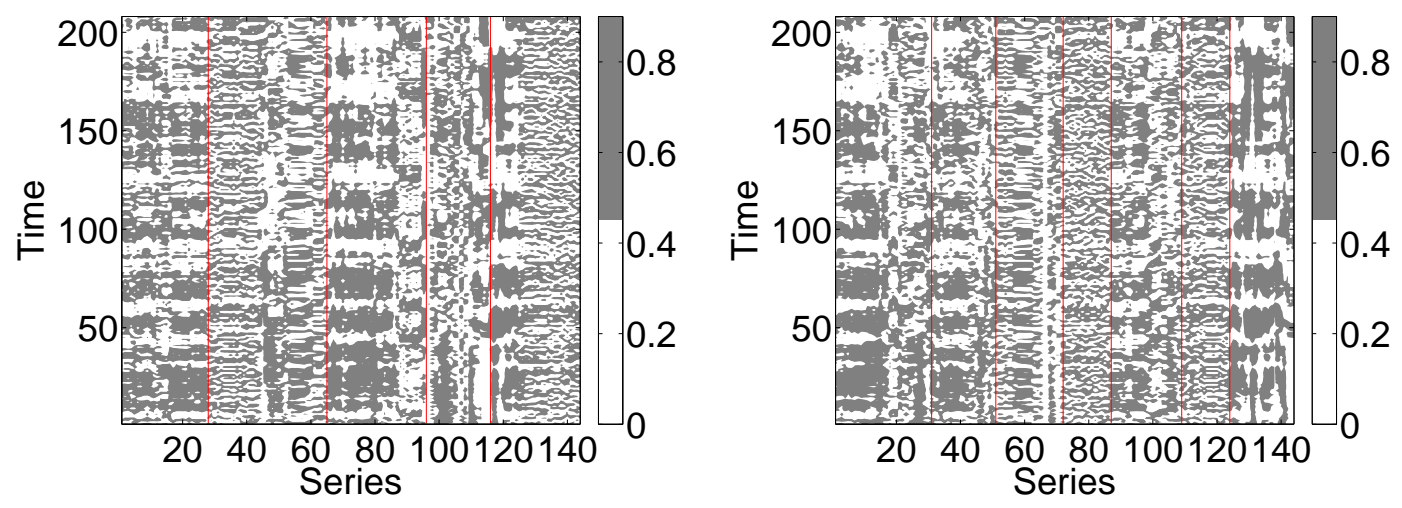

Figure S.5: Normal cumulative density function for the standardised series. The series are ordered by cluster label. We assume alternatively 5 (left) and 7 (right) clusters.

Figure S.6 shows the De Finetti's diagram of the two largest weights in the seven clusters for each of the variables to be predicted and a selection of horizons, $h=1,2,5$, using multivariate combinations and assuming $b_{k, i j}$ equal to the recursive $\log$ score for model $i$ in cluster $j$ when predicting the series $k$.

Figure S.8 shows a typical output of the model weights $\left(b_{k, i j}\right)$ in the seven clusters. There are large differences across clusters: the clusters 2, 4, 5 and 6 have few models with most of the weights; the other clusters, 1,3 and 7 , have more similar weights across models. This finding should be associated with the largest weights in Figure 3 for the clusters 2, 4, 5 and 6 and indicates that using recursive time-varying $b_{k, i j}$ weights within the clusters increases forecast accuracy for GDP growth relative to using equal weights. Figure S.8 also indicates that the weights within clusters are much more volatile than the cluster common component, indicating that individual model performances may change much over time even if information in a given clusters is stable. 

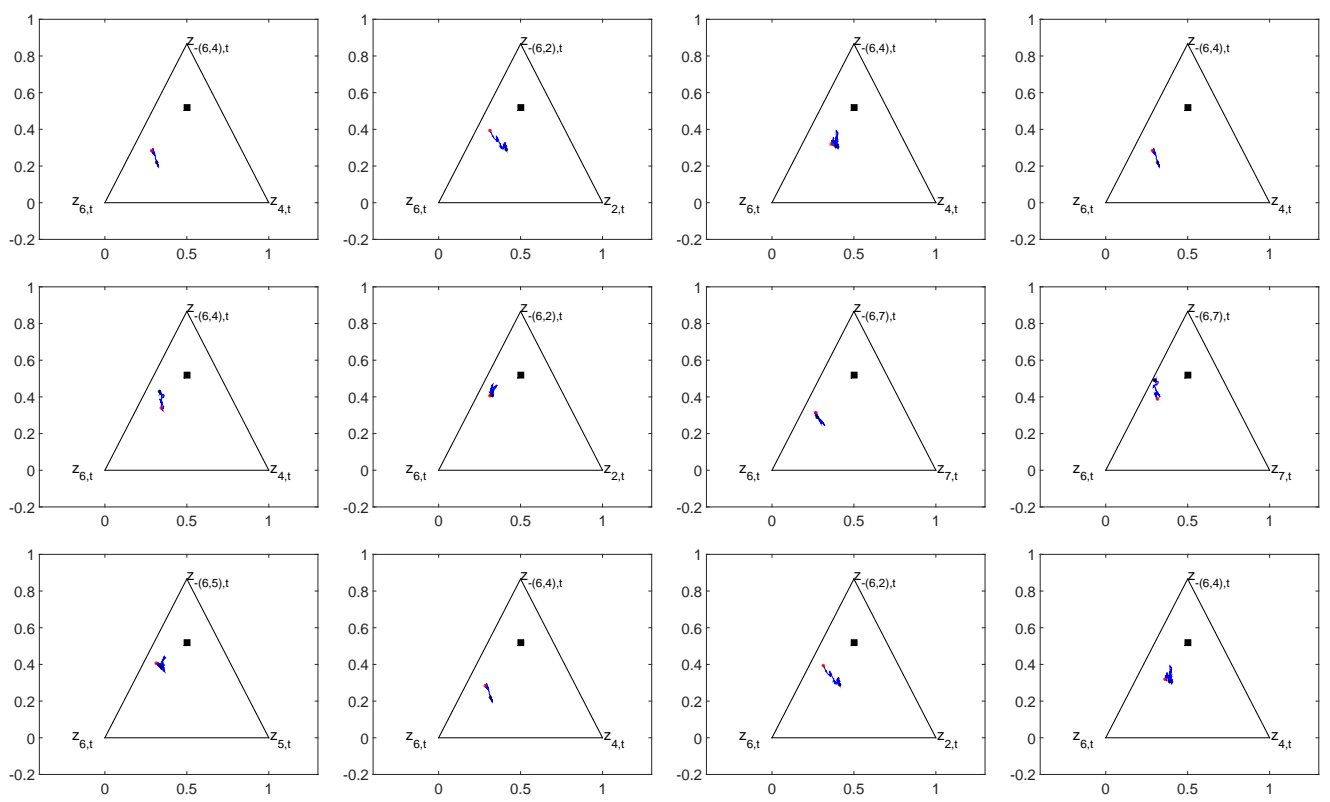

Figure S.6: De Finetti's diagrams for the dynamic comparison of the two largest weights. Rows: diagrams for the four series of interest (real GDP growth rate, GDP deflator, Treasury Bills, employment). Columns: forecast horizons (1, 3 and 5 quarters). In each plot the trajectory (blue line), the starting (red) and ending (black) points and the equal weight composition (square). 

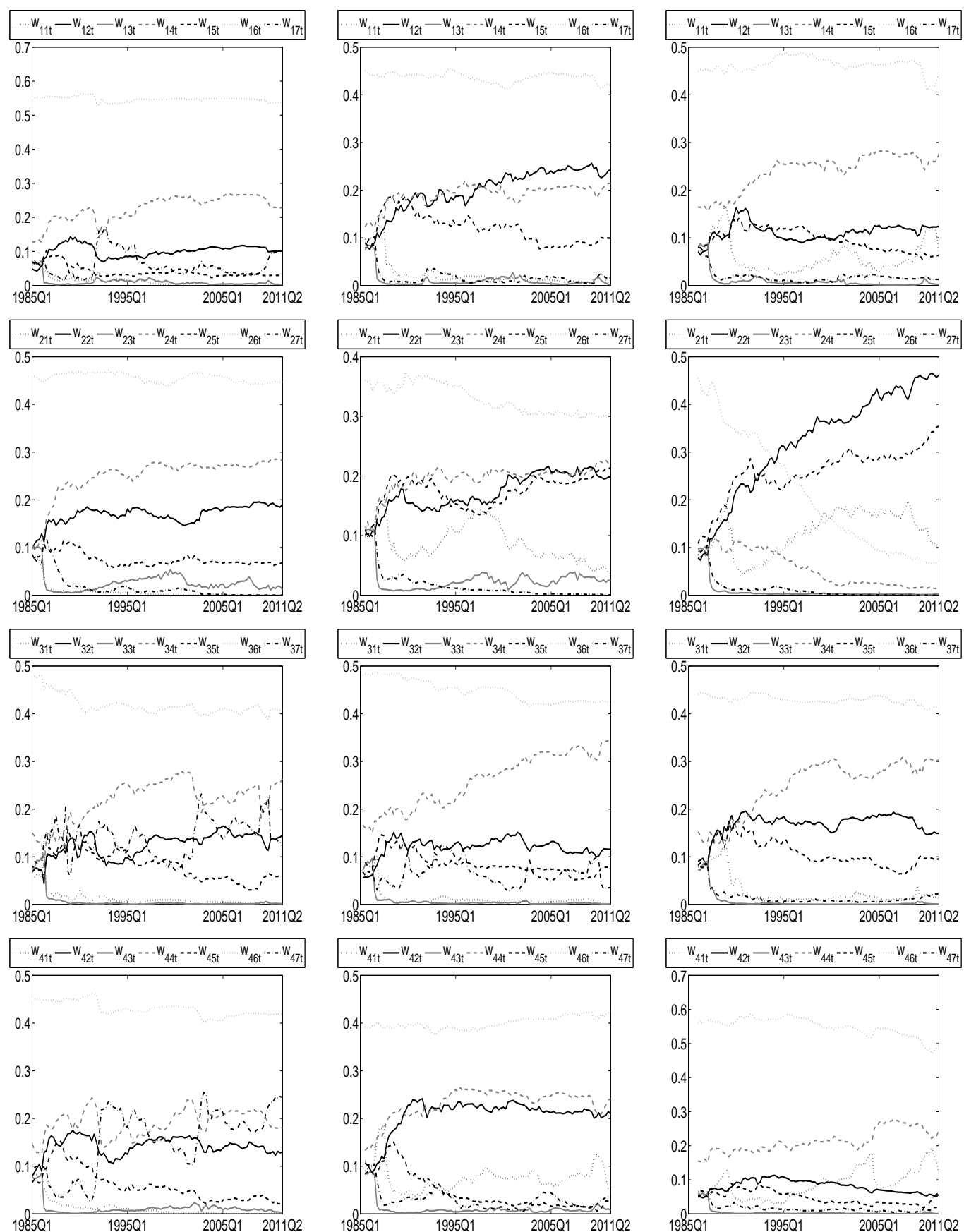

Figure S.7: In each plot the mean logistic-normal weights (different lines) for the univariate combination model are given. Rows: plot for the four series of interest (real GDP growth rate, GDP deflator, 3-month Treasury Bills, employment). Columns: forecast horizons (1, 3 and 5 quarters). 


\section{S.6 Additional details on the financial application}

Table S.4 reports the cross-section average statistics, together with statistics for the S\&P500. Some series have much lower average returns than the index and volatility higher than the index up to 400 times. Heterogeneity in skewness is also very evident with the series with lowest skewness equal to -42.5 and the one with highest skewness equal to 27.3 compared to a value equal to -0.18 for the index. Finally, maximum kurtosis is 200 times higher than the index value. The inclusion in our sample of the crisis period explains such differences, with some stocks that realized enormously negative returns in 2008 and impressive positive returns in 2009.

Figure S.9 presents the mean values of the predicted features $\boldsymbol{\psi}_{i t}$ which belong to the $j$-th cluster at each of the 784 vintages, labeled as $\mathbf{m}_{j t+1}$. The clusters for the Normal GARCH(1,1) models differ substantially in terms of predicted variance with cluster 1 having a rather low constant variance value over the entire period while cluster 2 has a variance more than double in size including a shock in the latter part of 2008 . For the $t$-GARCH $(1,1)$ model it is seen that cluster 3 has a relatively constant thick tail over the entire period while cluster 4 has an average value of 10 for the degrees of freedom and in the crisis period the density collapses to a normal density with degrees of freedom higher than 30 . In summary, The Lehman Brother effect is visible in the figure, with an increase of volatility in the normal cluster 2 and, interesting, an increase of the degrees of freedom in the $t$-cluster 4 .

\section{S.7 Computing time}

In this section we compare the computational speed of CPU with GPU in the implementation of our combination algorithm for both the financial and macro application. Whether CPU computing is standard in econometrics, GPU approach to computing has been received large attention in economics only recently. See, for example, Aldrich (2014) for a review, Geweke and Durham (2012) and Lee et al. (2010) for applications to Bayesian inference and Aldrich et al. (2011), Morozov and Mathur (2012) and Dziubinski and Grassi (2013) for solving DSGE models. 

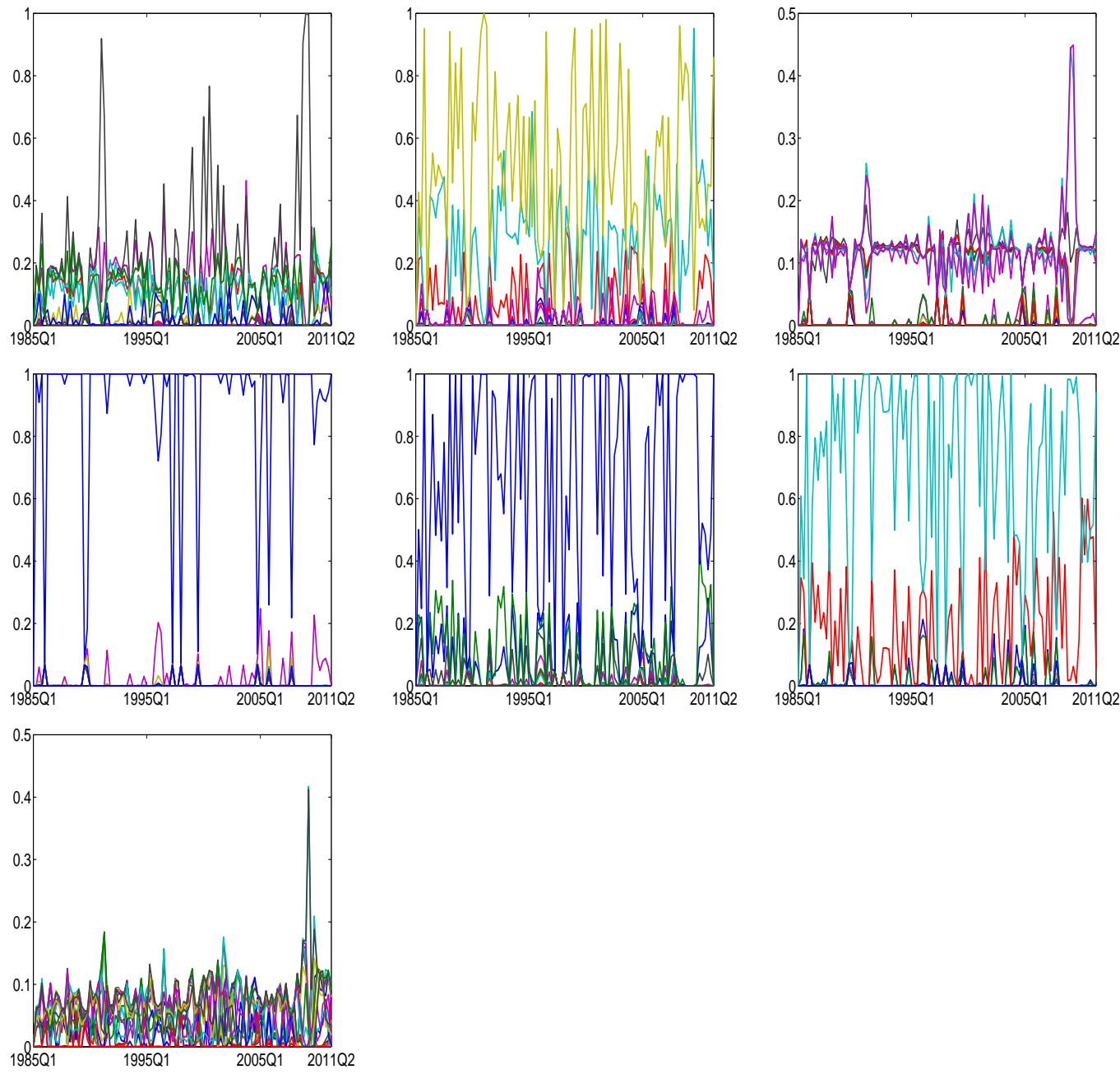

Figure S.8: The plots show the model weights $\left(b_{k, i j}\right)$ in each cluster $(i=j)$ when forecasting GDP growth $(k=1)$ at the 1-step ahead horizon. The first row refers to clusters 1,2 , and 3 ; the second row to clusters 4,5 , and 6 ; the last row to cluster 7 . 

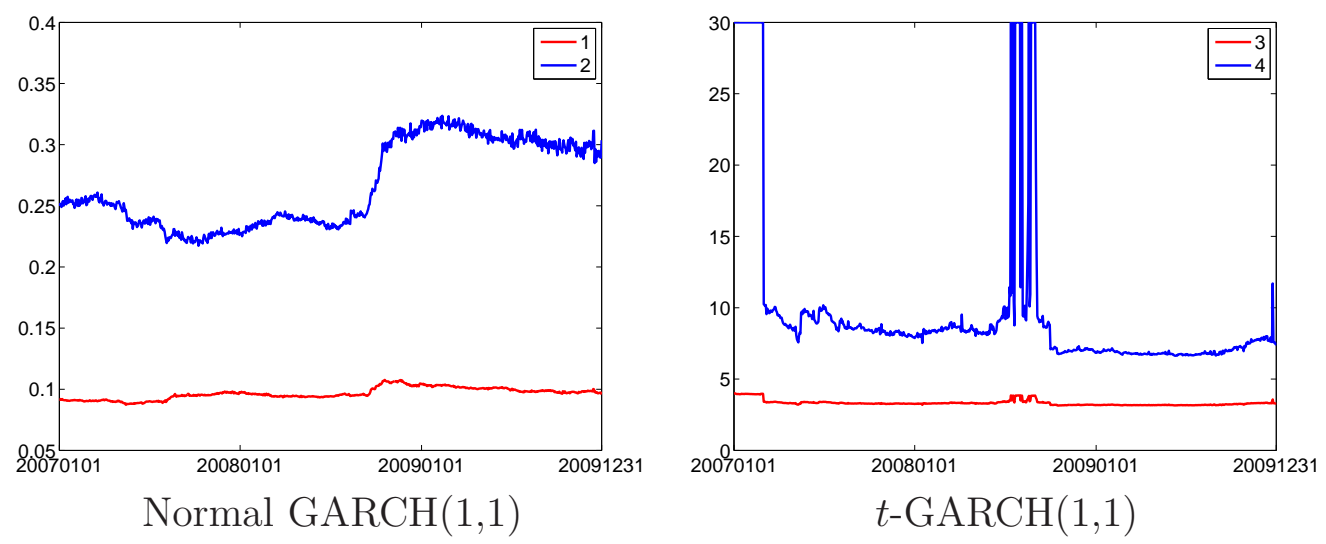

Figure S.9: The figures present the average variance of the predictions from the two clusters for the Normal GARCH(1,1) models based on low (cluster 1) and high (cluster 2 ) volatility in the left panel; and the average degree of freedom of the predictions from the two clusters for the $t$-GARCH(1,1) models based on low (cluster 3 ) and high (cluster 4) degrees of freedom in the right panel. The degrees of freedom are bounded to 30 .

The CPU and the GPU versions of the computer program are written in MATLAB, as described in Casarin et al. (2015). In the CPU setting, our test machine is a server with two Intel Xeon CPU E5-2667 v2 processors and a total of 32 core. In the first GPU setting, our test machine is a NVIDIA Tesla K40c GPU. The Tesla K40c card is with 12GB memory and 2880 cores and it is installed in the CPU server. In the second GPU setting, our test machine is a NVIDIA GeForce GTX 660 GPU card, which is a middle-level video card, with a total of 960 cores. The test machine is a desktop Windows 8 machine, has 16 GB of Ram and only requires a MATLAB parallel toolbox license.

We compare two sets of combination experiments, the density combination based on 4 clusters with equal weights within clusters and time-varying volatility, DCEW-SV, see Section 4.2, and the density combination with univariate combination based on 7 clusters with recursive log score weights within clusters, UDCLS7 $7^{4}$, see Section S.5, for an increasing number of particles $N$. In both sets of experiments we calculated, in seconds, the overall average execution time

\footnotetext{
${ }^{4}$ The case MCDCLS7 provide similar relative timing, in absolute terms a bit faster than the univariate ones.
} 


\begin{tabular}{lrrrr}
\hline & \multicolumn{3}{c}{ Subcomponents } & S\&P500 \\
\hline & Lower & Median & Upper & \\
Average & -0.002 & 0.000 & 0.001 & 0.000 \\
St dev & 0.016 & 0.035 & 0.139 & 0.019 \\
Skewness & -1.185 & 0.033 & 1.060 & -0.175 \\
Kurtosis & 8.558 & 16.327 & 65.380 & 9.410 \\
Min & -1.322 & -0.286 & -0.121 & -0.095 \\
Max & 0.122 & 0.264 & 1.386 & 0.110 \\
\hline
\end{tabular}

Table S.4: Average cross-section statistics for the 3712 individual stock daily log returns in our dataset for the sample 18 March 2002 to 31 December 2009. The columns "Lower", "Median" and "Upper" refer to the cross-section $10 \%$ lower quantile, median and 90\% upper quantile of the 3712 statistics in rows, respectively. The rows "Average", "St dev", "Skewness", "Kurtosis", "Min" and "Max" refers to sample average, sample standard deviation, sample skewness, sample kurtosis, sample minimum and sample maximum statistics, respectively. The column "S\&P500" reports the sample statistics for the aggregate S\&P500 log returns.

reported in Table S.5.

As the table shows, the CPU implementation is slower then the first GPU set-up in all cases. The NVIDIA Tesla K40c GPU provides gains in the order of magnitude from 2 to 4 times than the CPU. Very interestingly, even the second GPU set-up, which can be installed in a desktop machine, provides execution times comparable to the $\mathrm{CPU}$ in the financial applications and large gains in the macro applications. Therefore, the GPU environment seems the preferred one for our density combination problems and when the number of predictive density becomes very large a GPU server card gives the highest gains. 


\begin{tabular}{rrrrrrr}
\hline & \multicolumn{3}{c}{ DCEW-SV } & \multicolumn{3}{c}{ UDCLS7 } \\
Draws & 100 & 500 & 1000 & 100 & 500 & 1000 \\
\hline CPU & 1032 & 5047 & 10192 & 5124 & 25683 & 51108 \\
GPU 1 & 521 & 2107 & 4397 & 1613 & 6307 & 14017 \\
GPU 2 & 1077 & 5577 & 13541 & 2789 & 13895 & 27691 \\
\hline Ratio 1 & 1.98 & 2.39 & 2.32 & 3.18 & 4.07 & 3.65 \\
Ratio 2 & 0.96 & 0.90 & 0.75 & 1.84 & 1.85 & 1.85 \\
\hline
\end{tabular}

Table S.5: Observed total time (in seconds) and CPU/GPU ratios for the algorithm on CPU and GPU on different machines and with different numbers of particles. The CPU is a 32 core Intel Xeon CPU E5-2667 v2 two processors and the GPU1 is a NVIDIA Tesla K40c GPU and the GPU2 is a NVIDIA GeForce GTX 660. "Ratio 1 " refers to the CPU/GPU 1 ratio and "ratio 2" refers to the CPU/GPU 2 ratios. Number below 1 indicates the CPU is faster, number above one indicates that the GPU is faster. 


\section{References}

Aastveit, K. A., Gerdrup, K. R., Jore, A. S., and Thorsrud, L. A. (2014). Nowcasting GDP in real-time:A density combination approach. Journal of Business Economics \&5 Statistics, 32:48-68.

Aitchinson, J. and Shen, S. M. (1980). Logistic-normal distributions: Some properties and uses. Biometrika, 67:261-272.

Aldrich, E. M. (2014). Gpu computing in economics. In L., K. J. and Schmedders, K., editors, Handbook of Computational Economics, Vol. 3. Elsevier.

Aldrich, E. M., Fernández-Villaverde, J., Gallant, A. R., and Rubio Ramirez, J. F. (2011). Tapping the supercomputer under your desk: Solving dynamic equilibrium models with graphics processors. Journal of Economic Dynamics and Control, 35:386-393.

Amisano, G. and Giacomini, R. (2007). Comparing density forecasts via weighted likelihood ratio tests. Journal of Business \&6 Economic Statistics, 25:177-190.

Andrews, D. W. K. and Monahan, J. C. (1992). An improved heteroskedasticity and autocorrelation consistent covariance matrix estimator. Econometrica, 60:953-966.

Billio, M., Casarin, R., Ravazzolo, F., and van Dijk, H. K. (2013). Timevarying combinations of predictive densities using nonlinear filtering. Journal of Econometrics, 177:213-232.

Casarin, R., Grassi, S., Ravazzolo, F., and van Dijk, H. K. (2015). Parallel sequential Monte Carlo for efficient density combination: the DeCo Matlab toolbox. Jounal of Statistical Software, 68(3).

Casarin, R. and Marin, J. M. (2009). Online data processing: Comparison of Bayesian regularized particle filters. Electronic Journal of Statistics, 3:239258. 
Clark, T. E. and McCracken, M. W. (2011). Testing for unconditional predictive ability. In Clements, M. and Hendry, D., editors, Oxford Handbook of Economic Forecasting. Oxford University Press, Oxford.

Clark, T. E. and McCracken, M. W. (2015). Nested forecast model comparisons: a new approach to testing equal accuracy. Journal of Econometrics, 186:160177.

Clark, T. E. and Ravazzolo, F. (2015). The macroeconomic forecasting performance of autoregressive models with alternative specifications of timevarying volatility. Journal of Applied Econometrics, 30:551-575.

Diebold, F. X. and Mariano, R. S. (1995). Comparing predictive accuracy. Journal of Business \& Economic Stastistics, 13:253-263.

Dziubinski, M. P. and Grassi, S. (2013). Heterogeneous computing in economics: A simplified approach. Computational Economics, 43:485-495.

Favirar, R., Rebolledo, D., Chan, E., and Campbell, R. (2008). A parallel implementation of K-Means clustering on GPUs. Proceedings of 2008 International Conference on Parallel and Distributed Processing Techniques and Applications, 2:14-17.

Geweke, J. and Durham, G. (2012). Massively parallel sequential Monte Carlo for Bayesian inference. Working papers, National Bureau of Economic Research, Inc.

Gneiting, T. and Raftery, A. E. (2007). Strictly proper scoring rules, prediction, and estimation. Journal of the American Statistical Association, 102:359-378.

Gneiting, T. and Ranjan, R. (2013). Combining predicitve distributions. Electronic Journal of Statistics, 7:1747-1782.

Groen, J. J. J., Paap, R., and Ravazzolo, F. (2013). Real-time inflation forecasting in a changing world. Journal of Business 85 Economic Stastistics, $31: 29-44$. 
Hall, S. G. and Mitchell, J. (2007). Combining density forecasts. International Journal of Forecasting, 23:1-13.

Kascha, C. and Ravazzolo, F. (2010). Combining inflation density forecasts. Journal of Forecasting, 29:231-250.

Kitagawa, G. (1998). Self-organizing state space model. Journal of the American Statistical Association, 93:1203-1215.

Kitagawa, G. and Sato, S. (2001). Monte Carlo smoothing and self-organizing state-space model. In Doucet, A., de Freitas, N., and Gordon, N., editors, Sequential Monte Carlo Methods in Practice. Springer-Verlag.

Lee, A., Yau, C., Giles, M. B., Doucet, A., and Holmes, C. C. (2010). On the utility of graphic cards to perform massively parallel simulation with advanced Monte Carlo methods. Journal of Computational and Graphical Statistics, 19:769-789.

Liu, J. and West, M. (2001). Combined parameter and state estimation in simulation based filtering. In Doucet, A., de Freitas, N., and Gordon, N., editors, Sequential Monte Carlo Methods in Practice. Springer-Verlag.

Marcellino, M., Stock, J. H., and Watson, M. W. (2006). A comparison of direct and iterated multistep AR methods for forecasting macroeconomic time series. Journal of Econometrics, 135:499-526.

Mitchell, J. and Hall, S. G. (2005). Evaluating, comparing and combining density forecasts using the KLIC with an application to the Bank of England and NIESER "fan" charts of inflation. Oxford Bulletin of Economics and Statistics, 67:995-1033.

Morozov, S. and Mathur, S. (2012). Massively parallel computation using graphics processors with application to optimal experimentation in dynamic control. Computational Economics, 40:151-182.

Ravazzolo, F. and Vahey, S. V. (2014). Forecast densities for economic aggregates from disaggregate ensembles. Studies in Nonlinear Dynamics and Econometrics, 18:367-381. 
Stock, J. H. and Watson, W. M. (2005). Implications of dynamic factor models for VAR analysis. Technical report, NBER Working Paper No. 11467. 